\title{
The first Hochschild cohomology group of quantum matrices and the quantum special linear group
}

\author{
S. Launois and T. H. Lenagan*
}

\begin{abstract}
We calculate the first Hochschild cohomology group of quantum matrices, the quantum general linear group and the quantum special linear group in the generic case when the deformation parameter is not a root of unity. As a corollary, we obtain information about twisted Hochschild homology of these algebras.
\end{abstract}

Mathematics Subject Classification (2000). 16E40, 16W35, 17B37, 17B40, $20 \mathrm{G} 42$.

Keywords. Quantum matrices, quantum special linear group, derivation, Hochschild cohomology, twisted Hochschild homology.

\section{Introduction}

There has been interest recently in calculating Hochschild homology and cohomology for certain quantum groups and quantum algebras, see, for example, papers by Hadfield and Krähmer [6], [7], and Brown and Zhang [2]. In this paper, we begin to study the Hochschild cohomology of the algebra of quantum matrices, $\mathcal{O}_{q}\left(M_{n}\right)$, in the generic case where $q$ is not a root of unity. To be more specific, we calculate the first Hochschild cohomology, $\mathrm{HH}^{1}\left(\mathcal{O}_{q}\left(M_{n}\right)\right)$, of $\mathcal{O}_{q}\left(M_{n}\right)$ : in other words, we calculate the derivations of $\mathcal{O}_{q}\left(M_{n}\right)$. Once this has been done, we are also able to calculate $\mathrm{HH}^{1}$ for the quantum general linear group, $\mathcal{O}_{q}\left(\mathrm{GL}_{n}\right)$, and the quantum special linear group, $\mathcal{O}_{q}\left(\mathrm{SL}_{n}\right)$.

Alev and Chamarie [1] have calculated $\operatorname{HH}^{1}\left(\mathcal{O}_{q}\left(M_{2}\right)\right)$ directly by using the commutation relations for $\mathcal{O}_{q}\left(M_{2}\right)$. It seems impossible to follow this route in the general case: the commutation relations one would have to deal with are far too involved. Thus, we have taken another approach to the problem, by using Cauchon's theory of deleting derivations.

Even via this approach, the calculations are necessarily very technical. However, the idea is relatively easy to follow. The starting point is a result of Osborn and Passman

\footnotetext{
${ }^{*}$ This research was supported by a Marie Curie Intra-European Fellowship within the $6^{\text {th }}$ European Community Framework Programme and by Leverhulme Research Interchange Grant F/00158/X.
} 
[12] that describes the derivations of a quantum torus. In particular, they show that the first Hochschild cohomology group of the quantum torus with $n^{2}$ generators is a free module of rank $n^{2}$ over the centre of the quantum torus. The key to transfering this result to $\mathcal{O}_{q}\left(M_{n}\right)$ is Cauchon's theory of deleting derivations, introduced in [3], [4]. The algebra $\mathcal{O}_{q}\left(M_{n}\right)$ is presented in a natural way as an iterated Ore extension in $n^{2}$ steps. In $(n-1)^{2}$ of these steps a nontrivial skew derivation is involved. The quantum torus of rank $n^{2}$ is a localisation of a quantum affine space of dimension $n^{2}$. This quantum affine space is an iterated Ore extension in $n^{2}$ steps and no skew derivations are involved in any of the steps. Cauchon shows that one can construct a chain of algebras, starting from $\mathcal{O}_{q}\left(M_{n}\right)$ and finishing with a quantum affine space of dimension $n^{2}$. At each stage in the construction of this chain of algebras, the two adjacent algebras are equal up to the inversion of the powers of an element; and so information can be passed along the chain. However, at $(n-1)^{2}$ of the stages, the newly constructed algebra can be presented as an iterated Ore extension using one fewer skew derivation. This process can be reversed, and then at $(n-1)^{2}$ stages a skew derivation is re-introduced into the presentation of the algebra as an iterated Ore extension. Informally, in reintroducing a skew derivation to the presentation, one loses a derivation from the first Hochschild cohomology group. Thus, by the time one has re-introduced all $(n-1)^{2}$ skew derivations and recovered $\mathcal{O}_{q}\left(M_{n}\right)$, there remain $n^{2}-(n-1)^{2}=2 n-1$ derivations in $\operatorname{HH}^{1}\left(\mathcal{O}_{q}\left(M_{n}\right)\right)$; in other words, $\operatorname{HH}^{1}\left(\mathcal{O}_{q}\left(M_{n}\right)\right)$ is free of rank $2 n-1$ over the centre of $\mathcal{O}_{q}\left(M_{n}\right)$. The technical problems arise due to two main problems. First, the formulae involved in the deleting and re-introducing skew derivations process are awkward to deal with. Secondly, the centres change along the way.

In the last section, we apply our main result to compute the first Hochschild cohomology group of the quantum groups $\mathcal{O}_{q}\left(\mathrm{GL}_{n}\right)$ and $\mathcal{O}_{q}\left(\mathrm{SL}_{n}\right)$.

Regarding the Hochschild homology of $\mathcal{O}_{q}\left(\mathrm{SL}_{n}\right)$, Feng and Tsygan have shown [5] that $\mathrm{HH}_{k}\left(\mathcal{O}_{q}\left(\mathrm{SL}_{n}\right), \mathcal{O}_{q}\left(\mathrm{SL}_{n}\right)\right)=0$ for all $k \geq n$, whereas the global dimension of $\mathcal{O}_{q}\left(\mathrm{SL}_{n}\right)$ is $n^{2}-1$. In other words, there is a "dimension drop" phenomenon in the Hochschild homology of $\mathcal{O}_{q}\left(\mathrm{SL}_{n}\right)$. To deal with this problem, Hadfield and Krähmer [6], [7] have shown that one should use the twisted Hochschild homology defined by Kustermans, Murphy and Tuset [9] rather than classical Hochschild homology. The twisted Hochschild homology of $\mathcal{O}_{q}\left(\mathrm{SL}_{n}\right)$ depends on an automorphism of $\mathcal{O}_{q}\left(\mathrm{SL}_{n}\right)$. When $\sigma$ is the modular automorphism associated to the Haar functional of $\mathcal{O}_{q}\left(\mathrm{SL}_{n}\right)$ ([8], Section 11.3), Hadfield and Krähmer have shown that the twisted Hochschild homology group of degree $n^{2}-1$ is reduced to the base field $K$; that is, $\mathrm{HH}_{n^{2}-1}^{\sigma}\left(\mathcal{O}_{q}\left(\mathrm{SL}_{n}\right)\right)=K$, so that the "dimension drop" phenomenon disappears. This result was recently generalised to any connected complex semisimple algebraic group $G$ by Brown and Zhang [2]. In the last section of this paper, thanks to a (twisted) Poincaré duality between the twisted Hochschild homology associated to the modular automorphism and the Hochschild cohomology of $\mathcal{O}_{q}\left(\mathrm{SL}_{n}\right)$ [7], [14] we derive new 
information on the twisted Hochschild homology of $\mathcal{O}_{q}\left(\mathrm{SL}_{n}\right)$ : roughly speaking, we show that, when $G$ is a connected complex semisimple algebraic group of type $A$, the rank of the algebraic group $G$ appears as a twisted homological invariant of the quantised coordinate ring of $G$.

In an earlier paper [10] we have calculated the automorphism group of $\mathcal{O}_{q}\left(M_{m, n}\right)$ in the case that $m \neq n$. Partial results were obtained for the square case $\mathcal{O}_{q}\left(M_{n}\right)$, but technicalities prevented a resolution of the problem in this case. In a subsequent paper, we intend to use the results obtained in this paper to finish the calculation of the automorphism group of $\mathcal{O}_{q}\left(M_{n}\right)$.

\section{The deleting derivations algorithm in the algebra of quantum matrices}

In this section, we present briefly the deleting-derivations algorithm and use it to construct a tower of algebras from the algebra of quantum matrices to a quantum torus. This tower will be used in the next section to obtain the derivations of the algebra of quantum matrices from the derivations of the quantum torus.

1.1. The algebra of quantum matrices. Throughout this paper, we use the following conventions.

- The cardinality of a finite set $I$ is denoted by $|I|$.

- $\llbracket a, b \rrbracket:=\{i \in \mathbb{N} \mid a \leq i \leq b\}$.

- $K$ denotes a field of characteristic 0 and $K^{*}:=K \backslash\{0\}$.

- $q \in K^{*}$ is not a root of unity.

- $n$ denotes a positive integer with $n>1$.

- $R=\mathcal{O}_{q}\left(M_{n}\right)$ is the quantisation of the ring of regular functions on $n \times n$ matrices with entries in $K$; it is the $K$-algebra generated by the $n \times n$ indeterminates $Y_{i, \alpha}$, for $1 \leq i, \alpha \leq n$, subject to the following relations:

$$
\begin{aligned}
Y_{i, \beta} Y_{i, \alpha} & =q^{-1} Y_{i, \alpha} Y_{i, \beta} & & (\alpha<\beta), \\
Y_{j, \alpha} Y_{i, \alpha} & =q^{-1} Y_{i, \alpha} Y_{j, \alpha} & & (i<j), \\
Y_{j, \beta} Y_{i, \alpha} & =Y_{i, \alpha} Y_{j, \beta} & & (i<j, \alpha>\beta), \\
Y_{j, \beta} Y_{i, \alpha} & =Y_{i, \alpha} Y_{j, \beta}-\left(q-q^{-1}\right) Y_{i, \beta} Y_{j, \alpha} & & (i<j, \alpha<\beta) .
\end{aligned}
$$

It is well known that $R$ can be presented as an iterated Ore extension over $K$, with the generators $Y_{i, \alpha}$ adjoined in lexicographic order. Thus the ring $R$ is a Noetherian domain; its skew-field of fractions is denoted by $F$.

1.2. The deleting derivations algorithm and some related algebras. First, recall, see [4], that the theory of deleting derivations can be applied to the iterated Ore extension $R=K\left[Y_{1,1}\right]\left[Y_{1,2} ; \sigma_{1,2}\right] \ldots\left[Y_{n, n} ; \sigma_{n, n}, \delta_{n, n}\right]$ (where the indices are increasing 
for the lexicographic order $\leq$ ). The corresponding deleting derivations algorithm is called the standard deleting derivations algorithm. Before recalling its construction, we need to introduce some notation.

- The lexicographic ordering on $\mathbb{N}^{2}$ is denoted by $\leq_{s}$. This order is often referred to as the standard ordering on $\mathbb{N}^{2}$. Recall that $(i, \alpha) \leq_{s}(j, \beta)$ if and only if $[(i<j)$ or $(i=j$ and $\alpha \leq \beta)]$.

- Set $E=\left(\llbracket 1, n \rrbracket^{2} \cup\{(n, n+1)\}\right) \backslash\{(1,1)\}$.

- Let $(j, \beta) \in E$ with $(j, \beta) \neq(n, n+1)$. The least element (relative to $\left.\leq_{s}\right)$ of the set $\left\{(i, \alpha) \in E \mid(j, \beta)<_{s}(i, \alpha)\right\}$ is denoted by $(j, \beta)^{+}$.

As described in [4], the standard deleting derivations algorithm constructs, for each $r \in E$, a family $\left\{Y_{i, \alpha}^{(r)}\right\}$, for $(i, \alpha) \in \llbracket 1, n \rrbracket^{2}$, of elements of $F:=\operatorname{Frac}(R)$, defined as follows:

(1) If $r=(n, n+1)$, then $Y_{i, \alpha}^{(n, n+1)}=Y_{i, \alpha}$ for all $(i, \alpha) \in \llbracket 1, n \rrbracket^{2}$.

(2) Assume that $r=(j, \beta)<_{s}(n, n+1)$ and that the $Y_{i, \alpha}^{\left(r^{+}\right)}$for $(i, \alpha) \in \llbracket 1, n \rrbracket^{2}$ are already constructed. Then it follows from [3], Théorème 3.2.1, that each $Y_{j, \beta}^{\left(r^{+}\right)} \neq 0$ and that, for all $(i, \alpha) \in \llbracket 1, n \rrbracket^{2}$, we have

$$
Y_{i, \alpha}^{(r)}= \begin{cases}Y_{i, \alpha}^{\left(r^{+}\right)}-Y_{i, \beta}^{\left(r^{+}\right)}\left(Y_{j, \beta}^{\left(r^{+}\right)}\right)^{-1} Y_{j, \alpha}^{\left(r^{+}\right)} & \text {if } i<j \text { and } \alpha<\beta, \\ Y_{i, \alpha}^{\left(r^{+}\right)} & \text {otherwise. }\end{cases}
$$

As in [3], for all $(j, \beta) \in E$, the subalgebra of $\operatorname{Frac}(R)$ generated by the indeterminates $Y_{i, \alpha}^{(j, \beta)}$, with $(i, \alpha) \in \llbracket 1, n \rrbracket^{2}$, is denoted by $R^{(j, \beta)}$. Also, $\bar{R}$ denotes the subalgebra of $\operatorname{Frac}(R)$ generated by the indeterminates obtained at the end of this algorithm; that is, $\bar{R}$ is the subalgebra of $\operatorname{Frac}(R)$ generated by the $T_{i, \alpha}:=Y_{i, \alpha}^{(1,2)}$ for each $(i, \alpha) \in \llbracket 1, n \rrbracket^{2}$.

Recall [3], Theorem 3.2.1, that, for all $(j, \beta) \in E$, the algebra $R^{(j, \beta)}$ can be presented as an iterated Ore extension over $K$, with the generators $Y_{i, \alpha}^{(j, \beta)}$ adjoined in lexicographic order. Thus the algebra $R^{(j, \beta)}$ is a Noetherian domain.

For all $(j, \beta) \in E$, the multiplicative system generated by the indeterminates $T_{i, \alpha}$, for $(i, \alpha) \geq(j, \beta)$ with $i>1$ and $\alpha>1$, is denoted by $S_{(j, \beta)}$. As $T_{i, \alpha}=Y_{i, \alpha}^{(j, \beta)}$, for all $(i, \alpha) \geq(j, \beta)$ with $i>1$ and $\alpha>1$, the set $S_{(j, \beta)}$ is a multiplicative system of regular elements of $R^{(j, \beta)}$. Moreover, the $T_{i, \alpha}$ such that $(i, \alpha) \geq(j, \beta)$ with $i>1$ and $\alpha>1$ are normal in $R^{(j, \beta)}$. Hence, $S_{(j, \beta)}$ is an Ore set in $R^{(j, \beta)}$; so that one can form the localisation

$$
U_{(j, \beta)}:=R^{(j, \beta)} S_{(j, \beta)}^{-1} .
$$

Clearly, the set of monomials of the form $\left(Y_{1,1}^{(j, \beta)}\right)^{\gamma_{1,1}}\left(Y_{1,2}^{(j, \beta)}\right)^{\gamma_{1,2}} \ldots\left(Y_{n, n}^{(j, \beta)}\right)^{\nu_{n, n}}$, with $\gamma_{i, \alpha} \in \mathbb{N}$ if $(i, \alpha)<(j, \beta)$ or $i=1$ or $\alpha=1$, and $\gamma_{i, \alpha} \in \mathbb{Z}$ otherwise, is a PBW basis of $U_{(j, \beta)}$. 
Further, recall from [4], Theorem 2.2.1, that $\Sigma_{(j, \beta)}:=\left\{\left(T_{j, \beta}\right)^{k} \mid k \in \mathbb{N}\right\}$ is an Ore set in both $R^{(j, \beta)}$ and $R^{(j, \beta)^{+}}$, and that

$$
R^{(j, \beta)} \Sigma_{(j, \beta)}^{-1}=R^{(j, \beta)^{+}} \Sigma_{(j, \beta)}^{-1} .
$$

Hence, we obtain the following result.

Lemma 1.1. $R^{(j, 1)}=R^{(j, 2)}$ and $U_{(j, 1)}=U_{(j, 2)}$.

Let $\beta>1$. Then $R^{(j, \beta)} \Sigma_{(j, \beta)}^{-1}=R^{(j, \beta)^{+}} \Sigma_{(j, \beta)}^{-1}$ and $U_{(j, \beta)}=U_{(j, \beta)}+\Sigma_{(j, \beta)}^{-1}$.

Let $N \in \mathbb{N}^{*}$ and let $\Lambda=\left(\Lambda_{i, j}\right)$ be a multiplicatively antisymmetric $N \times N$ matrix over $K^{*}$; that is, $\Lambda_{i, i}=1$ and $\Lambda_{j, i}=\Lambda_{i, j}^{-1}$ for all $i, j \in \llbracket 1, N \rrbracket$. The corresponding quantum affine space is denoted by $K_{\Lambda}\left[T_{1}, \ldots, T_{N}\right]$; that is, $K_{\Lambda}\left[T_{1}, \ldots, T_{N}\right]$ is the $K$-algebra generated by the $N$ indeterminates $T_{1}, \ldots, T_{N}$ subject to the relations $T_{i} T_{j}=\Lambda_{i, j} T_{j} T_{i}$ for all $i, j \in \llbracket 1, N \rrbracket$. In [4, Section 2.2], Cauchon has shown that $\bar{R}$ can be viewed as the quantum affine space generated by the indeterminates $T_{i, \alpha}$ for $(i, \alpha) \in \llbracket 1, n \rrbracket^{2}$, subject to the following relations:

$$
\begin{aligned}
& T_{i, \beta} T_{i, \alpha}=q^{-1} T_{i, \alpha} T_{i, \beta} \quad(\alpha<\beta), \\
& T_{j, \alpha} T_{i, \alpha}=q^{-1} T_{i, \alpha} T_{j, \alpha} \quad(i<j), \\
& T_{j, \beta} T_{i, \alpha}=T_{i, \alpha} T_{j, \beta} \quad(i<j, \alpha>\beta), \\
& T_{j, \beta} T_{i, \alpha}=T_{i, \alpha} T_{j, \beta} \quad(i<j, \alpha<\beta) .
\end{aligned}
$$

Hence, $\bar{R}=K_{\Lambda}\left[T_{1,1}, T_{1,2}, \ldots, T_{n, n}\right]$, where $\Lambda$ denotes the $n^{2} \times n^{2}$ matrix defined as follows. Set

$$
A:=\left(\begin{array}{rrrrr}
0 & 1 & 1 & \ldots & 1 \\
-1 & 0 & 1 & \ldots & 1 \\
\vdots & \ddots & \ddots & \ddots & \vdots \\
-1 & \ldots & -1 & 0 & 1 \\
-1 & \ldots & \ldots & -1 & 0
\end{array}\right) \in \mathcal{M}_{n}(\mathbb{Z})
$$

and

$$
B:=\left(\begin{array}{rrrrr}
A & I & I & \ldots & I \\
-I & A & I & \ldots & I \\
\vdots & \ddots & \ddots & \ddots & \vdots \\
-I & \ldots & -I & A & I \\
-I & \ldots & \ldots & -I & A
\end{array}\right) \in \mathcal{M}_{n^{2}}(\mathbb{Z})
$$

where $I$ denotes the identity matrix of $\mathcal{M}_{n}(\mathbb{Z})$. Then $\Lambda$ is the $n^{2} \times n^{2}$ matrix whose entries are defined by $\Lambda_{k, l}=q^{b_{k, l}}$ for all $k, l \in \llbracket 1, n^{2} \rrbracket$.

Now observe that

$$
U_{(2,2)}=K_{\Lambda}\left[T_{1,1}, T_{1,2}, \ldots, T_{1, n}, T_{2,1}, T_{2,2}^{ \pm 1}, \ldots, T_{2, n}^{ \pm 1}, \ldots, T_{n, 1}, T_{n, 2}^{ \pm 1}, \ldots, T_{n, n}^{ \pm 1}\right]
$$


In other words,

$$
U_{(2,2)}=\bar{R} S^{-1},
$$

where $S=S_{(2,2)}$ is the multiplicative system generated by the $T_{i, \alpha}$ with $i>1$ and $\alpha>1$.

In order to investigate the Lie algebra of derivations of $R$, we also need to introduce the following algebras.

For all $(j, \beta) \in \llbracket 1, n \rrbracket^{2}$ with $j=1$ or $\beta=1$, the multiplicative system generated by those $T_{i, \alpha}$ such that $(i, \alpha)>(j, \beta)$ and either $i=1$ or $\alpha=1$ is denoted by $\delta_{(j, \beta)}$. Clearly, $\diamond_{(j, \beta)}$ is an Ore set in $U_{(2,2)}$. Set

$$
V_{(j, \beta)}:=U_{(2,2)} 8_{(j, \beta)}^{-1},
$$

and observe that $V_{(n, 1)}=U_{(2,2)}$.

As the set of monomials $T_{1,1}^{\gamma_{1,1}} T_{1,2}^{\gamma_{1,2}} \ldots T_{n, n}^{\gamma_{n, n}}$, with $\gamma_{i, \alpha} \in \mathbb{N}$ if $i=1$ or $\alpha=1$, and $\gamma_{i, \alpha} \in \mathbb{Z}$ otherwise, is a PBW basis of $U_{(2,2)}$, it is easy to check that the set of monomials $T_{1,1}^{\gamma_{1,1}} T_{1,2}^{\gamma_{1,2}} \ldots T_{n, n}^{\gamma_{n, n}}$, with $\gamma_{i, \alpha} \in \mathbb{N}$ if $(i, \alpha) \leq(j, \beta)$ and either $i=1$ or $\alpha=1$, and $\gamma_{i, \alpha} \in \mathbb{Z}$ otherwise, is a PBW basis of $V_{(j, \beta)}$

Finally, set $V_{(1,0)}:=P(\Lambda)$, where $P(\Lambda)$ denotes the quantum torus associated to the quantum affine space $\bar{R}$; that is, the localisation of $\bar{R}$ with respect of the multiplicative system generated by all the $T_{i, \alpha}$. Recall that the set of monomials $\left\{T_{1,1}^{\gamma_{1,1}} T_{1,2}^{\gamma_{1,2}} \ldots T_{n, n}^{\gamma_{n, n}}\right\}$, with $\gamma_{i, \alpha} \in \mathbb{Z}$, forms a PBW basis of $P(\Lambda)$.

Our proof will use the tower of algebras

$$
\begin{gathered}
R=U_{(n, n+1)} \subset U_{(n, n)} \subset \cdots \subset U_{(2,3)} \subset U_{(2,2)}=V_{(n, 1)} \subset V_{(n-1,1)} \\
\subset \cdots \subset V_{(2,1)} \subset V_{(1, n)} \subset \cdots \subset V_{(1,0)}=P(\Lambda) .
\end{gathered}
$$

1.3. Quantum minors and the centres of $\mathcal{O}_{q}\left(M_{n}\right), P(\Lambda)$ and $U_{(2,2)}$. The algebra $\mathcal{O}_{q}\left(M_{n}\right)$ has a special element, the quantum determinant, denoted by $\operatorname{det}_{q}$, and defined by

$$
\operatorname{det}_{q}:=\sum_{\sigma}(-q)^{l(\sigma)} Y_{1, \sigma(1)} \ldots Y_{n, \sigma(n)},
$$

where the sum is taken over the permutations of $\{1, \ldots, n\}$ and $l(\sigma)$ is the usual length function on such permutations. The quantum determinant is a central element of $\mathcal{O}_{q}\left(M_{n}\right)$, see, for example, [13], Theorem 4.6.1. If $I$ and $\Gamma$ are $t$-element subsets of $\{1, \ldots, n\}$, then the quantum determinant of the subalgebra of $\mathcal{O}_{q}\left(M_{n}\right)$ generated by $Y_{i, \alpha}$, with $i \in I$ and $\alpha \in \Gamma$, is denoted by $[I \mid \Gamma]$. The elements $[I \mid \Gamma]$ are the quantum minors of $\mathcal{O}_{q}\left(M_{n}\right)$.

In order to describe the centres of $P(\Lambda)$ and $U_{(2,2)}$, we introduce the following quantum minors of $R$.

For $1 \leq i \leq 2 n-1$, let $b_{i}$ be the quantum minor defined as follows:

$$
b_{i}:= \begin{cases}{[1, \ldots, i \mid n-i+1, \ldots, n]} & \text { if } 1 \leq i \leq n, \\ {[i-n+1, \ldots, n \mid 1, \ldots, 2 n-i]} & \text { if } n<i \leq 2 n-1 .\end{cases}
$$


For convenience, we set $b_{0}=b_{2 n}=1$. Note that these $b_{i}$ are a priori elements of $R$. However, it turns out that they also belong to the quantum torus $P(\Lambda)$, as the following result shows.

Lemma 1.2. For $1 \leq i \leq 2 n-1$, we have

$$
b_{i}= \begin{cases}T_{1, n-i+1} T_{2, n-i+2} \ldots T_{i, n} & \text { if } 1 \leq i \leq n, \\ T_{i-n+1,1} T_{i-n+2,2} \ldots T_{n, 2 n-i} & \text { if } n \leq i \leq 2 n-1 .\end{cases}
$$

Proof. This follows from [4], Proposition 5.2.1 (see also [10], Lemma 2.2).

The centre of an algebra $A$ is denoted by $Z(A)$. Set $\Delta_{i}:=b_{i} b_{n+i}^{-1}$ for all $i \in$ $\{1, \ldots, n\}$. Notice that $\Delta_{n}=\operatorname{det}_{q}$.

It follows from Lemma 1.2 that the $\Delta_{i}$ belong to the quantum torus $P(\Lambda)$ : in fact, the $\Delta_{i}$ are also central. The following result is established in [10], Theorem 2.4.

Proposition 1.3. $Z(P(\Lambda))=K\left[\Delta_{1}^{ \pm 1}, \ldots, \Delta_{n}^{ \pm 1}\right]$.

It is useful to record for later use the expression for the $\Delta_{i}$ in terms of the $T_{i, \alpha}$.

Lemma 1.4. $\Delta_{i}=T_{1, n-i+1} T_{2, n-i+2} \ldots T_{i, n} T_{i+1,1}^{-1} T_{i+2,2}^{-1} \ldots T_{n, n-i}^{-1}$, for $1 \leq i \leq n$.

Proof. This follows easily from Lemma 1.2, noting the commutation relations between the $T_{i, \alpha}$.

We finish this section by describing the centre of the algebra $U_{(2,2)}$. First, observe that $Z\left(U_{(2,2)}\right) \subseteq Z(P(\Lambda))=K\left[\Delta_{1}^{ \pm 1}, \ldots, \Delta_{n}^{ \pm 1}\right]$, since $P(\Lambda)$ is a localisation of $U_{(2,2)}$. Next, by using the PBW-basis of $U_{(2,2)}$ together with the expressions for the $\Delta_{i}$ as products of certain $T_{i, \alpha}$ coming from Lemma 1.4, we obtain the following result.

Lemma 1.5. $Z\left(U_{(2,2)}\right)=K\left[\Delta_{n}\right]=K\left[\operatorname{det}_{q}\right]$.

\section{Derivations}

Recall that $R$ denotes the algebra of $n \times n$ generic quantum matrices. Our aim in this section is to investigate $\operatorname{Der}(R)$, the Lie algebra of derivations of $R$.

Let $D$ be a derivation of $R$.

First, as there exists a multiplicative system $\Sigma$ of $R$ such that $R \Sigma^{-1}=P(\Lambda)=$ $V_{(1,0)}$, see [3], Theorem 3.3.1, the derivation $D$ extends (uniquely) to a derivation of the quantum torus $P(\Lambda)$. It follows from [12], Corollary 2.3, that $D$ can be written as

$$
D=\operatorname{ad}_{x}+\theta,
$$


where $x \in P(\Lambda)=V_{(1,0)}$ and $\theta$ is a derivation of $P(\Lambda)$ such that $\theta\left(T_{i, \alpha}\right)=z_{i, \alpha} T_{i, \alpha}$ with $z_{i, \alpha} \in Z(P(\Lambda))$ for all $(i, \alpha) \in \llbracket 1, n \rrbracket^{2}$.

For $\underline{\gamma} \in \mathbb{Z}^{n^{2}}$, set

$$
T^{\underline{\gamma}}:=T_{1,1}^{\gamma_{1,1}} T_{1,2}^{\gamma_{1,2}} \ldots T_{n, n}^{\gamma_{n, n}} .
$$

As the set of monomials $\left\{T^{\underline{\gamma}}\right\}_{\underline{\gamma} \in \mathbb{Z}^{n^{2}}}$ forms a PBW basis of $P(\Lambda)$, one can write

$$
x=\sum_{\underline{\gamma} \in \mathcal{E}} c_{\underline{\gamma}} T^{\underline{\gamma}},
$$

where $\mathcal{E}$ is a finite subset of $\mathbb{Z}^{n^{2}}$ and $c_{\underline{\gamma}} \in K$. Moreover, as $\operatorname{ad}_{x}=\operatorname{ad}_{x+z}$ for all $z \in Z(P(\Lambda))$, one can assume that, for all $\underline{\gamma} \in \mathcal{E}$, the monomial $T^{\underline{\gamma}}$ does not belong to $Z(P(\Lambda))$.

Next recall that an element $y=\sum_{\underline{\gamma} \in \mathbb{Z}^{2}} y_{\underline{\gamma}} T^{\underline{\gamma}} \in P(\Lambda)$ is central if and only if $T^{\underline{\gamma}} \in Z(P(\Lambda))$ for each $\underline{\gamma} \in \mathbb{Z}^{n^{2}}$ such that $y_{\underline{\gamma}} \neq 0$. Denote by $\mathcal{F}$ the set of all $\gamma \in \mathbb{Z}^{n^{2}}$ such that $T^{\underline{\gamma}} \in Z(P(\Lambda))$. Then, for all $(i, \alpha) \in \llbracket 1, n \rrbracket^{2}$, we can write $z_{i, \alpha}$ in the form

$$
z_{i, \alpha}=\sum_{\underline{\gamma} \in \mathscr{F}} z_{i, \alpha, \underline{\gamma}} T^{\underline{\gamma}}
$$

with $z_{i, \alpha, \underline{\gamma}} \in K$.

Lemma 2.1. Let $0 \leq \beta \leq n$. Then $x \in V_{(1, \beta)}$.

Proof. The proof is by induction on $\beta$. The case $\beta=0$ follows from the above discussion, because $V_{(1,0)}=P(\Lambda)$. Hence, assume that $\beta \geq 1$.

It follows from the inductive hypothesis that

$$
x=\sum_{\underline{\gamma} \in \mathcal{E}} c_{\underline{\gamma}} T^{\underline{\gamma}},
$$

where $\mathcal{E}$ is a finite subset of the set $\left\{\gamma \in \mathbb{Z}^{n^{2}} \mid \gamma_{1,1} \geq 0, \ldots, \gamma_{1, \beta-1} \geq 0\right.$ and $T^{\underline{\gamma}} \notin$ $Z(P(\Lambda))\}$. We need to prove that $\gamma_{1, \beta} \geq 0$.

Observe that, by construction, $V_{(1, \beta)}$ is obtained from $R$ by a sequence of localisations. Thus, $D$ extends to a derivation of $V_{(1, \beta)}$. Let $(i, \alpha) \neq(1, \beta)$. Then $D\left(T_{i, \alpha}\right) \in V_{(1, \beta)}$, since $T_{i, \alpha} \in V_{(1, \beta)}$; that is,

$$
x T_{i, \alpha}-T_{i, \alpha} x+z_{i, \alpha} T_{i, \alpha} \in V_{(1, \beta)} .
$$

Set

$$
x_{+}:=\sum_{\substack{\underline{\underline{\gamma}} \in \mathcal{E} \\ \gamma_{1, \beta} \geq 0}} c_{\underline{\gamma}} T^{\underline{\underline{\nu}}}, \quad x_{-}=\sum_{\substack{\underline{\gamma} \in \mathcal{E} \\ \gamma_{1, \beta}<0}} c_{\underline{\gamma}} T^{\underline{\gamma}} .
$$


We need to prove that $x_{-}=0$.

It follows from (2) that

$$
u:=x_{-} T_{i, \alpha}-T_{i, \alpha} x_{-}+z_{i, \alpha} T_{i, \alpha} \in V_{1, \beta} .
$$

Now

$$
\begin{aligned}
u= & \sum_{\substack{\underline{\gamma} \in \mathcal{E} \\
\gamma_{1, \beta}<0}}\left(q^{-\exp (i, \alpha, \underline{\gamma},+)}-q^{-\exp (i, \alpha, \underline{\gamma},-)}\right) c_{\underline{\gamma}} T^{\underline{\gamma}+\varepsilon_{i, \alpha}} \\
& +\sum_{\underline{\gamma} \in \mathcal{F}} q^{-\exp (i, \alpha, \underline{\gamma},+)} z_{i, \alpha, \underline{\gamma}} T^{\underline{\gamma}+\varepsilon_{i, \alpha}},
\end{aligned}
$$

where

$\exp (i, \alpha, \underline{\gamma},-):=\sum_{k=1}^{i-1} \gamma_{k, \alpha}+\sum_{k=1}^{\alpha-1} \gamma_{i, k}, \exp (i, \alpha, \underline{\gamma},+):=\sum_{k=i+1}^{n} \gamma_{k, \alpha}+\sum_{k=\alpha+1}^{n} \gamma_{i, k}$

and $\varepsilon_{i, \alpha}$ is the element of $\mathbb{Z}^{n^{2}}$ that has 1 in the $(i, \alpha)$ position and zero elsewhere. As we have assumed that the monomial $T^{\underline{\gamma}}$ does not belong to $Z(P(\Lambda))$ for all $\underline{\gamma} \in \mathcal{E}$, we have

$$
\underline{\gamma}+\varepsilon_{i, \alpha} \neq \underline{\gamma}^{\prime}+\varepsilon_{i, \alpha} \quad \text { for all } \underline{\gamma} \in \mathcal{E} \text { and for all } \underline{\gamma}^{\prime} \in \mathcal{F} \text {. }
$$

Hence (3) gives the expression of $u$ in the PBW basis of $P(\Lambda)$.

On the other hand, as $u$ belongs to $V_{(1, \beta)}$, we obtain

$$
u=\sum_{\underline{\gamma} \in \mathcal{E}^{\prime}} x_{\underline{\gamma}} T^{\underline{\gamma}}
$$

where $\mathcal{E}^{\prime}$ is a finite subset of $\left\{\gamma \in \mathbb{Z}^{n^{2}} \mid \gamma_{1,1} \geq 0, \ldots, \gamma_{1, \beta} \geq 0\right\}$.

Comparing the two expressions of $u$ in the PBW basis of $P(\Lambda)$ leads to $q^{-\exp (i, \alpha, \underline{\gamma},+)}-q^{-\exp (i, \alpha, \underline{\gamma},-)}=0$ for all $\underline{\gamma} \in \mathcal{E}$ such that $\gamma_{1, \beta}<0$ and $c_{\underline{\gamma}} \neq 0$. Hence

$$
x_{-} T_{i, \alpha}-T_{i, \alpha} x_{-}=\sum_{\substack{\underline{\underline{\underline{x}}} \in \mathcal{E} \\ \gamma_{1, \beta}<0}}\left(q^{-\exp (i, \alpha, \underline{\gamma},+)}-q^{-\exp (i, \alpha, \underline{\gamma},-)}\right) c_{\underline{\gamma}} T^{\underline{\gamma}+\varepsilon_{i, \alpha}}=0
$$

for all $(i, \alpha) \neq(j, \beta)$. In other words, $x_{-}$commutes with those $T_{i, \alpha}$ such that $(i, \alpha) \neq(1, \beta)$.

Now recall from Lemma 1.4 that

$$
\Delta_{n+1-\beta}=T_{1, \beta} T_{2, \beta+1} \ldots T_{n+1-\beta, n} T_{n+2-\beta, 1}^{-1} T_{n+3-\beta, 2}^{-1} \ldots T_{n, \beta-1}^{-1}
$$


is central in $P(\Lambda)$. Hence, $x_{-}$also commutes with $T_{1, \beta}$. This implies that $x_{-} \in$ $Z(P(\Lambda))$; so that $x_{-}$can be written as

$$
x_{-}=\sum_{\underline{\gamma} \in \mathscr{F}} d_{\underline{\gamma}} T^{\underline{\gamma}} .
$$

Hence $x_{-}=0$, because $\mathcal{E} \cap \mathcal{F}=\emptyset$; so that $x=x_{+} \in V_{(1, \beta)}$, as desired.

The following result is proved by using similar arguments.

Lemma 2.2. Let $2 \leq j \leq n$. Then $x \in V_{(j, 1)}$. In particular, $x \in V_{(n, 1)}=U_{(2,2)}$.

The derivation $D$ of $R$ extends to a derivation of $U_{(2,2)}$, since $U_{(2,2)}$ is obtained from $R$ by a sequence of localisations; so $D\left(T_{i, \alpha}\right) \in U_{(2,2)}$ for all $(i, \alpha) \in \llbracket 1, n \rrbracket^{2}$. Hence

$$
x T_{i, \alpha}-T_{i, \alpha} x+z_{i, \alpha} T_{i, \alpha}=D\left(T_{i, \alpha}\right) \in U_{(2,2)} .
$$

As we have proved that $x \in U_{(2,2)}$, this implies that $z_{i, \alpha} T_{i, \alpha} \in U_{(2,2)}$ for all $(i, \alpha) \in$ $\llbracket 1, n \rrbracket^{2}$.

If $i \geq 2$ and $\alpha \geq 2$ then $z_{i, \alpha} \in U_{(2,2)} \cap Z(P(\Lambda))=Z\left(U_{(2,2)}\right)$ because $T_{i, \alpha}$ is invertible in $U_{(2,2)}$. However, $Z\left(U_{(2,2)}\right)=K\left[\Delta_{n}\right]$ by Lemma 1.5 ; so $z_{i, \alpha} \in K\left[\Delta_{n}\right] \subseteq$ $R$ in this case.

In the other cases, at this stage in the proof we can only prove a weaker result.

Assume that $i=1$ and $\alpha>1$. Then $z_{1, \alpha} T_{1, \alpha} \in U_{(2,2)}$. On the other hand, as $z_{1, \alpha}$ belongs to the centre of the quantum torus $P(\Lambda)$, one can write $z_{1, \alpha}$ as follows:

$$
z_{1, \alpha}=P\left(\Delta_{1}, \ldots, \Delta_{n}\right) \in K\left[\Delta_{1}^{ \pm 1}, \ldots, \Delta_{n}^{ \pm 1}\right] .
$$

Now, using the expressions of the $\Delta_{i}$ as products of $T_{j, \beta}^{ \pm 1}$ coming from Lemma 1.4, we obtain

$$
z_{1, \alpha}=\sum_{\underline{\gamma} \in \mathcal{Z}} z_{1, \alpha, \underline{\gamma}} T^{\underline{\gamma}}
$$

where $\mathcal{Z}$ denotes the set of those $\underline{\gamma}=\left(\gamma_{1,1}, \gamma_{1,2}, \ldots, \gamma_{n, n}\right) \in \mathbb{Z}^{n^{2}}$ such that

1) $\gamma_{1,1}=\gamma_{2,2}=\cdots=\gamma_{n, n}$,

2) $\gamma_{1, \beta}=\gamma_{2, \beta+1}=\cdots=\gamma_{n-\beta+1, n}=-\gamma_{n-\beta+2,1}=\cdots=-\gamma_{n, \beta-1}$ for all $\beta \in \llbracket 1, n \rrbracket$,

and $z_{1, \alpha, \underline{\gamma}} \in K$ for all $\underline{\gamma} \in \mathcal{Z}$.

Hence

$$
z_{1, \alpha} T_{1, \alpha}=\sum_{\underline{\gamma} \in \mathcal{Z}} z_{i, \alpha, \underline{\gamma}}^{\prime} T^{\underline{\gamma}+\varepsilon_{1, \alpha}} \in U_{(2,2)},
$$


where $z_{1, \alpha, \gamma}^{\prime}=q^{\bullet} z_{1, \alpha, \underline{\gamma}}$ for all $\underline{\gamma} \in \mathcal{Z}$. As the monomials $T_{1,1}^{\gamma_{1,1}} T_{1,2}^{\gamma_{1,2}} \ldots T_{n, n}^{\gamma_{n, n}}$, where $\gamma_{j, \beta} \in \mathbb{N}$ when either $j=1$ or $\beta=1$, and $\gamma_{j, \beta} \in \mathbb{Z}$ otherwise, form a PBW basis of $U_{(2,2)}$, we obtain $z_{1, \alpha, \underline{\gamma}}^{\prime}=0$ whenever

either $\gamma_{1,1}<0, \quad$ or $\quad \gamma_{1, \beta} \neq 0$ for some $\beta \neq 1, \alpha, \quad$ or $\quad \gamma_{1, \alpha} \notin\{-1,0\}$.

Hence we easily deduce from (4) and Lemma 1.4 that there exist polynomials $P_{1, \alpha}$, $Q_{1, \alpha} \in K\left[\Delta_{n}\right]$ such that

$$
z_{1, \alpha}=Q_{1, \alpha}\left(\Delta_{n}\right) \Delta_{n+1-\alpha}^{-1}+P_{1, \alpha}\left(\Delta_{n}\right) .
$$

Similar computations for $z_{i, 1}$, for $i>1$, and for $z_{1,1}$ lead to the following result.

Proposition 2.3. 1) $x \in U_{(2,2)}$. that

2) Let $(i, \alpha) \in \llbracket 1, n \rrbracket^{2}$. Then there exist polynomials $P_{i, \alpha}, Q_{i, \alpha} \in K\left[\Delta_{n}\right]$ such

$$
z_{i, \alpha}= \begin{cases}Q_{i, \alpha}\left(\Delta_{n}\right) \Delta_{n+1-\alpha}^{-1}+P_{i, \alpha}\left(\Delta_{n}\right) & \text { if } i=1, \\ Q_{i, \alpha}\left(\Delta_{n}\right) \Delta_{i-1}+P_{i, \alpha}\left(\Delta_{n}\right) & \text { if } \alpha=1, \\ P_{i, \alpha}\left(\Delta_{n}\right) & \text { otherwise. }\end{cases}
$$

(Here we use the convention $\Delta_{0}=b_{0} b_{n}^{-1}=\Delta_{n}^{-1}$.)

Next, we have to deal with a second kind of localisation that involves inverting an element which is not normal. This is done in several steps.

Lemma 2.4. 1) $x \in U_{(2,3)}$.

2) $z_{1,1}+z_{2,2}=z_{1,2}+z_{2,1}$.

3) $z_{1,1}, z_{1,2}, z_{2,1}$ and $z_{2,2}$ belong to $Z(R)=K\left[\Delta_{n}\right]$.

4) $D\left(Y_{i, \alpha}^{(2,3)}\right)=\operatorname{ad}_{x}\left(Y_{i, \alpha}^{(2,3)}\right)+z_{i, \alpha} Y_{i, \alpha}^{(2,3)}$ for all $(i, \alpha) \in \llbracket 1, n \rrbracket^{2}$.

Proof. Step 1: we prove that $x \in U_{(2,3)}$.

In order to simplify the notation, set $Z_{i, \alpha}:=Y_{i, \alpha}^{(2,3)}$ for all $(i, \alpha) \in \llbracket 1, n \rrbracket^{2}$. Moreover, for all $\underline{\gamma} \in \mathcal{E}:=\mathbb{N}^{n} \times\left(\mathbb{N} \times \mathbb{Z}^{n-1}\right) \times \cdots \times\left(\mathbb{N} \times \mathbb{Z}^{n-1}\right) \subset \mathbb{Z}^{n^{2}}$, set

$$
Z^{\underline{\gamma}}:=Z_{1,1}^{\gamma_{1,1}} Z_{1,2}^{\gamma_{1,2}} \ldots Z_{n, n}^{\gamma_{n, n}} .
$$

It follows from Proposition 2.3 that $x$ belongs to $U_{(2,2)}$. Using the notation of the previous section, it follows from Lemma 1.1 that

$$
U_{(2,2)}=U_{(2,3)} \Sigma_{(2,2)}^{-1},
$$

so that $x$ can be written as

$$
x=\sum_{\underline{\gamma} \in \mathcal{E}} c_{\underline{\gamma}} Z \underline{\underline{\gamma}},
$$


with $c_{\underline{\gamma}} \in K$. Set

$$
x_{+}:=\sum_{\substack{\underline{\gamma} \in \mathcal{E} \\ \gamma_{2,2} \geq 0}} c_{\underline{\gamma}} Z \underline{\underline{\gamma}}, \quad x_{-}:=\sum_{\substack{\underline{\gamma} \in \mathcal{E} \\ \gamma_{2,2}<0}} c_{\underline{\gamma}} Z \underline{\underline{\gamma}},
$$

with $c_{\underline{\gamma}} \in K$. Assume that $x_{-} \neq 0$.

Denote by $B$ the subalgebra of $U_{(2,2)}$ generated by the $Z_{i, \alpha}$ with $(i, \alpha) \neq(2,2)$ and the $Z_{i, \alpha}^{-1}$ with $i \geq 2$ and $\alpha \geq 2$ but $(i, \alpha) \neq(2,2)$. Hence $U_{(2,2)}=U_{(2,3)} \Sigma_{(2,2)}^{-1}$ is a left $B$-module with basis $\left\{Z_{2,2}^{l}\right\} l \in \mathbb{Z}$, so that there are elements $b_{l} \in B$ such that

$$
x_{-}=\sum_{l=l_{0}}^{-1} b_{l} Z_{2,2}^{l}
$$

with $l_{0}<0$ and $b_{l_{0}} \neq 0$. (Observe that this makes sense because we have assumed that $x_{-} \neq 0$.)

The derivation $D$ of $R$ extends to a derivation of $U_{(2,3)}$, since $U_{(2,3)}$ is obtained from $R$ by a sequence of localisations; so $D\left(Z_{1,1}\right) \in U_{(2,3)}$. Now $Z_{1,1}=T_{1,1}+$ $T_{1,2} T_{2,2}^{-1} T_{2,1}=T_{1,1}+Z_{1,2} Z_{2,2}^{-1} Z_{2,1}$, so that

$$
x_{-} Z_{1,1}-Z_{1,1} x_{-}+z_{1,1} Z_{1,1}+\left(z_{1,2}+z_{2,1}-z_{1,1}-z_{2,2}\right) Z_{1,2} Z_{2,2}^{-1} Z_{2,1} \in U_{(2,3)} .
$$

Now

$$
Z_{2,2}^{-k} Z_{1,1}=Z_{1,1} Z_{2,2}^{-k}+q\left(q^{2 k}-1\right) Z_{1,2} Z_{2,1} Z_{2,2}^{-k-1}
$$

for each positive integer $k$. Hence

$$
\begin{aligned}
x_{-} Z_{1,1}- & Z_{1,1} x_{-}+z_{1,1} Z_{1,1}+\left(z_{1,2}+z_{2,1}-z_{1,1}-z_{2,2}\right) Z_{1,2} Z_{2,2}^{-1} Z_{2,1} \\
= & \sum_{l=l_{0}}^{-1} b_{l}^{\prime} Z_{2,2}^{l}+\sum_{l=l_{0}}^{-1} q\left(q^{-2 l}-1\right) b_{l} Z_{1,2} Z_{2,1} Z_{2,2}^{l-1} \\
& \quad-\left(z_{1,2}+z_{2,1}-z_{1,1}-z_{2,2}\right) Z_{1,2} Z_{2,2}^{-1} Z_{2,1}+z_{1,1} Z_{1,1} \in U_{(2,3)} .
\end{aligned}
$$

It follows from Proposition 2.3 that $z_{1,1} \operatorname{det}_{q}, z_{1,2} b_{n-1}$ and $z_{2,1} b_{n+1}$ belong to $R \subset U_{(2,3)}$. On the other hand, it follows from [4], Proposition 5.2.1, that $\operatorname{det}_{q}=\left(Z_{1,1} Z_{2,2}-q Z_{1,2} Z_{2,1}\right) Z_{3,3} \ldots Z_{n, n}$, while $b_{n-1}=Z_{1,2} Z_{2,3} \ldots Z_{n-1, n}$ and $b_{n+1}=Z_{2,1} \ldots Z_{n, n-1}$. Hence each of $z_{1,1}\left(Z_{1,1} Z_{2,2}-q Z_{1,2} Z_{2,1}\right), z_{1,2} Z_{1,2}$ and $z_{2,1} Z_{2,1}$ belong to $U_{(2,3)}$. As $z_{2,2} \in R$, by Proposition 2.3, we obtain

$$
\left(z_{1,2}+z_{2,1}-z_{1,1}-z_{2,2}\right) Z_{1,2} Z_{2,1}\left(Z_{1,1} Z_{2,2}-q Z_{1,2} Z_{2,1}\right) \in U_{(2,3)} .
$$


Multiplying (6) on the right by $\left(Z_{1,1} Z_{2,2}-q Z_{1,2} Z_{2,1}\right) Z_{2,2}$ leads to

$$
\begin{aligned}
& \sum_{l=l_{0}}^{-1} b_{l}^{\prime}\left(Z_{1,1} Z_{2,2}-q Z_{1,2} Z_{2,1}\right) Z_{2,2}^{l+1} \\
& \quad+\sum_{l=l_{0}}^{-1} q\left(q^{-2 l}-1\right) b_{l} Z_{1,2} Z_{2,1}\left(Z_{1,1} Z_{2,2}-q Z_{1,2} Z_{2,1}\right) Z_{2,2}^{l} \in U_{(2,3)} .
\end{aligned}
$$

In other words,

$$
\sum_{l=l_{0}+1}^{1} b_{l}^{\prime \prime} Z_{2,2}^{l}-q^{2}\left(q^{-2 l_{0}}-1\right) b_{l_{0}} Z_{1,2}^{2} Z_{2,1}^{2} Z_{2,2}^{l_{0}} \in U_{(2,3)} .
$$

As $U_{(2,3)}$ is a left $B$-module with basis $\left\{Z_{2,2}^{l}\right\}_{l \in \mathbb{N}}$, this implies that $b_{l_{0}}=0$, a contradiction. Hence $x_{-}=0$ and $x=x_{+} \in U_{(2,3)}$, as desired.

Step 2: we prove that $z_{1,1}+z_{2,2}=z_{1,2}+z_{2,1}$.

As $x_{-}=0$ and $z_{1,1}\left(Z_{1,1} Z_{2,2}-q Z_{1,2} Z_{2,1}\right) \in U_{(2,3)}$, we deduce from (5) that

$$
y:=\left(z_{1,2}+z_{2,1}-z_{1,1}-z_{2,2}\right) Z_{1,2} Z_{2,1}\left(Z_{1,1} Z_{2,2}-q Z_{1,2} Z_{2,1}\right) \in U_{(2,3)} Z_{2,2} \text {. }
$$

So $y$ is an element of $U_{(2,3)}$ which $q$-commutes with $Z_{1,1}$ and which belongs to $U_{(2,3)} Z_{2,2}$. We show next that this forces $y=0$, so that $z_{1,1}+z_{2,2}=z_{1,2}+z_{2,1}$, as desired.

Since $U_{(2,3)}$ is a left $B$-module with basis $\left\{Z_{2,2}^{l}\right\}_{l \in \mathbb{N}}$, one can write $y=\sum_{l \in \mathbb{N}} y_{l} Z_{2,2}^{l}$ with $y_{l} \in B$ equal to zero except for at most a finite number of them. As $y$ belongs to $U_{(2,3)} Z_{2,2}$, it is easy to show that $y_{0}=0$, so that

$$
y=\sum_{l \in \mathbb{N}, l \neq 0} y_{l} Z_{2,2}^{l} .
$$

On the other hand, as $y q$-commutes with $Z_{1,1}$, there exists $a \in \mathbb{Z}$ such that $Z_{1,1} y=$ $q^{a} y Z_{1,1}$. In other words,

$$
\sum_{l \in \mathbb{N}, l \neq 0} Z_{1,1} y_{l} Z_{2,2}^{l}=\sum_{l \in \mathbb{N}, l \neq 0} q^{a} y_{l} Z_{2,2}^{l} Z_{1,1}
$$

As $Z_{2,2}^{l} Z_{1,1}=Z_{1,1} Z_{2,2}^{l}+q\left(q^{-2 l}-1\right) Z_{1,2} Z_{2,1} Z_{2,2}^{l-1}$ for all positive integers $l$, we get

$$
\begin{aligned}
\sum_{l \in \mathbb{N}, l \neq 0} Z_{1,1} y_{l} Z_{2,2}^{l}= & \sum_{l \in \mathbb{N}, l \neq 0} q^{a} y_{l} Z_{1,1} Z_{2,2}^{l} \\
& +\sum_{l \in \mathbb{N}, l \neq 0} q^{a+1}\left(q^{-2 l}-1\right) y_{l} Z_{1,2} Z_{2,1} Z_{2,2}^{l-1}
\end{aligned}
$$


Assume that $y \neq 0$ and let $l_{0}$ be minimal such that $y_{l_{0}} \neq 0$. Observe that $l_{0} \geq 1$. As $U_{(2,3)}$ is a left $B$-module with basis $\left\{Z_{2,2}^{l}\right\} l \in \mathbb{N}$, we deduce from the previous equality that we should have $0=q^{a+1}\left(q^{-2 l_{0}}-1\right) y_{l_{0}} Z_{1,2} Z_{2,1}$, a contradiction since $l_{0} \geq 1$ and $q$ is not a root of unity. So $y=0$, as desired.

Step 3: we prove that $z_{1,1}, z_{1,2}, z_{2,1}$ and $z_{2,2}$ belong to $Z(R)$.

It follows from Proposition 2.3 that

$$
\begin{array}{ll}
z_{1,1}=Q_{1,1} \Delta_{n}^{-1}+P_{1,1}\left(\Delta_{n}\right), & z_{1,2}=Q_{1,2}\left(\Delta_{n}\right) \Delta_{n-1}^{-1}+P_{1,2}\left(\Delta_{n}\right), \\
z_{2,1}=Q_{2,1}\left(\Delta_{n}\right) \Delta_{1}+P_{2,1}\left(\Delta_{n}\right), & z_{2,2}=P_{2,2}\left(\Delta_{n}\right),
\end{array}
$$

where $Q_{1,1} \in K$ and $Q_{i, \alpha}, P_{i, \alpha} \in K\left[\Delta_{n}\right]$ otherwise. As $z_{1,1}+z_{2,2}=z_{1,2}+z_{2,1}$, we obtain

$$
\begin{aligned}
Q_{1,1} \Delta_{n}^{-1}+ & P_{1,1}\left(\Delta_{n}\right)+P_{2,2}\left(\Delta_{n}\right) \\
& =Q_{1,2}\left(\Delta_{n}\right) \Delta_{n-1}^{-1}+Q_{2,1}\left(\Delta_{n}\right) \Delta_{1}+P_{1,2}\left(\Delta_{n}\right)+P_{2,1}\left(\Delta_{n}\right) .
\end{aligned}
$$

Recalling that the monomials $\Delta_{1}^{i_{1}} \ldots \Delta_{n}^{i_{n}}$, with $i_{k} \in \mathbb{Z}$, are linearly independent, we obtain

$$
Q_{1,1}=Q_{1,2}\left(\Delta_{n}\right)=Q_{2,1}\left(\Delta_{n}\right)=0,
$$

so that $z_{1,1}=P_{1,1}\left(\Delta_{n}\right), z_{1,2}=P_{1,2}\left(\Delta_{n}\right), z_{2,1}=P_{2,1}\left(\Delta_{n}\right)$. Hence $z_{1,1}, z_{1,2}$ and $z_{2,1}$ belong to $K\left[\Delta_{n}\right]=Z(R)$, and we have already observed that $z_{2,2}=P_{2,2}\left(\Delta_{n}\right) \in$ $K\left[\Delta_{n}\right]=Z(R)$.

Step 4: we prove that $D\left(Z_{i, \alpha}\right)=\operatorname{ad}_{x}\left(Z_{i, \alpha}\right)+z_{i, \alpha} Z_{i, \alpha}$ for all $(i, \alpha) \in \llbracket 1, n \rrbracket^{2}$.

If $(i, \alpha) \neq(1,1)$, then $Z_{i, \alpha}=T_{i, \alpha}$ and so the result is obvious.

Next, consider the case $(i, \alpha)=(1,1)$. Note that $Z_{1,1}=T_{1,1}+T_{1,2} T_{2,2}^{-1} T_{2,1}$. Hence,

$$
\begin{aligned}
D\left(Z_{1,1}\right)= & D\left(T_{1,1}+T_{1,2} T_{2,2}^{-1} T_{2,1}\right) \\
= & \operatorname{ad}_{x}\left(T_{1,1}\right)+z_{1,1} T_{1,1} \\
& \quad+\operatorname{ad}_{x}\left(T_{1,2} T_{2,2}^{-1} T_{2,1}\right)+\left(z_{1,2}-z_{2,2}+z_{2,1}\right) T_{1,2} T_{2,2}^{-1} T_{2,1} \\
= & \operatorname{ad}_{x}\left(Z_{1,1}\right)+z_{1,1} Z_{1,1}+\left(z_{1,2}-z_{2,2}+z_{2,1}-z_{1,1}\right) T_{1,2} T_{2,2}^{-1} T_{2,1} .
\end{aligned}
$$

Now it follows from the second step that $z_{1,2}-z_{2,2}+z_{2,1}-z_{1,1}=0$. Hence,

$$
D\left(Z_{1,1}\right)=\operatorname{ad}_{x}\left(Z_{1,1}\right)+z_{1,1} Z_{1,1},
$$

as desired.

The next two lemmas continue the process of descending down the tower of algebras (1). Although the proofs superficially look the same as the proof of the previous lemma, there are subtle differences in each proof; so we find it necessary to include the full proofs. 
Lemma 2.5. Let $\beta \in \llbracket 2, n \rrbracket$.

1) $x \in U_{(2, \beta+1)}$. (Here we use the convention $U_{(2, n+1)}:=U_{(3,1)}$.)

2) For all $\alpha<\beta$, we have $z_{1, \alpha}+z_{2, \beta}=z_{1, \beta}+z_{2, \alpha}$.

3) $z_{1, \beta} \in Z(R)$.

4) $D\left(Y_{i, \alpha}^{(2, \beta+1)}\right)=\operatorname{ad}_{x}\left(Y_{i, \alpha}^{(2, \beta+1)}\right)+z_{i, \alpha} Y_{i, \alpha}^{(2, \beta+1)}$ for all $(i, \alpha) \in \llbracket 1, n \rrbracket^{2}$. (Here we use the convention $Y_{i, \alpha}^{(2, n+1)}:=Y_{i, \alpha}^{(3,1)}$.)

Proof. The proof is by induction on $\beta$. The case $\beta=2$ has been dealt with in the previous lemma. Now, assume that $\beta \geq 2$ and that the lemma has been proved for $\beta$. In order to simplify the notation, set $Z_{i, \alpha}:=Y_{i, \alpha}^{(2, \beta+1)}$ for all $(i, \alpha) \in \llbracket 1, n \rrbracket^{2}$. Moreover, for all $\underline{\gamma} \in \mathcal{E}:=\mathbb{N}^{n} \times\left(\mathbb{N}^{\beta-1} \times \mathbb{Z}^{n+1-\beta}\right) \times\left(\mathbb{N} \times \mathbb{Z}^{n-1}\right) \times \cdots \times\left(\mathbb{N} \times \mathbb{Z}^{n-1}\right)$, set

$$
Z^{\underline{\gamma}}:=Z_{1,1}^{\gamma_{1,1}} Z_{1,2}^{\gamma_{1,2}} \ldots Z_{n, n}^{\gamma_{n, n}} .
$$

We now proceed in five steps.

Step 1: we prove that $x \in U_{(2, \beta+1)}$.

It follows from the inductive hypothesis that $x$ belongs to $U_{(2, \beta)}$. Using the notation of previous sections, we have:

$$
U_{(2, \beta)}=U_{(2, \beta+1)} \Sigma_{2, \beta}^{-1},
$$

so that $x$ can be written as

$$
x=\sum_{\underline{\gamma} \in \mathcal{E}} c_{\underline{\gamma}} Z \underline{\underline{\gamma}}
$$

with $c_{\underline{\gamma}} \in K$. Set

$$
x_{+}:=\sum_{\substack{\underline{\underline{\gamma}} \in \mathcal{E} \\ \gamma_{2}, \beta \geq 0}} c_{\underline{\gamma}} Z^{\underline{\gamma}}, \quad x_{-}:=\sum_{\substack{\underline{\underline{\gamma}} \in \mathcal{E} \\ \gamma_{2, \beta}<0}} c_{\underline{\gamma}} Z^{\underline{\gamma}} .
$$

Assume that $x_{-} \neq 0$.

Denote by $B$ the subalgebra of $U_{(2, \beta)}$ generated by the $Z_{i, \alpha}$ with $(i, \alpha) \neq(2, \beta)$ and the $Z_{i, \alpha}^{-1}$ with $i \geq 2$ and $\alpha \geq 2$ but $(i, \alpha)>(2, \beta)$. Then $U_{(2, \beta)}=U_{(2, \beta+1)} \Sigma_{2, \beta}^{-1}$ is a left $B$-module with basis $\left\{Z_{2, \beta}^{l}\right\} l \in \mathbb{Z}$, so that there are elements $b_{l} \in B$ such that

$$
x_{-}=\sum_{l=l_{0}}^{-1} b_{l} Z_{2, \beta}^{l}
$$

with $l_{0}<0$ and $b_{l_{0}} \neq 0$. (Observe that this makes sense because we have assumed that $x_{-} \neq 0$.) 
The derivation $D$ of $R$ extends to a derivation of $U_{(2, \beta+1)}$, since $U_{(2, \beta+1)}$ is obtained from $R$ by a sequence of localisations; so $D\left(Z_{1, \beta-1}\right) \in U_{(2, \beta+1)}$. This implies that

$$
\begin{aligned}
x_{-} Z_{1, \beta-1} & -Z_{1, \beta-1} x_{-}+z_{1, \beta-1} Z_{1, \beta-1} \\
& +\left(z_{1, \beta}+z_{2, \beta-1}-z_{1, \beta-1}-z_{2, \beta}\right) Z_{1, \beta} Z_{2, \beta}^{-1} Z_{2, \beta-1} \in U_{(2, \beta+1)} .
\end{aligned}
$$

Now

$$
Z_{2, \beta}^{-k} Z_{1, \beta-1}=Z_{1, \beta-1} Z_{2, \beta}^{-k}+q\left(q^{2 k}-1\right) Z_{1, \beta} Z_{2, \beta-1} Z_{2, \beta}^{-k-1}
$$

for each positive integer $k$. Hence,

$$
\begin{aligned}
x_{-} Z_{1, \beta-1}- & Z_{1, \beta-1} x_{-}+z_{1, \beta-1} Z_{1, \beta-1} \\
& +\left(z_{1, \beta}+z_{2, \beta-1}-z_{1, \beta-1}-z_{2, \beta}\right) Z_{1, \beta} Z_{2, \beta}^{-1} Z_{2, \beta-1} \\
= & \sum_{l=l_{0}}^{-1} b_{l}^{\prime} Z_{2, \beta}^{l}+\sum_{l=l_{0}}^{-1} q\left(q^{-2 l}-1\right) b_{l} Z_{1, \beta} Z_{2, \beta-1} Z_{2, \beta}^{l-1} \\
& -\left(z_{1, \beta}+z_{2, \beta-1}-z_{1, \beta-1}-z_{2, \beta}\right) Z_{1, \beta} Z_{2, \beta}^{-1} Z_{2, \beta-1} \\
& +z_{1, \beta-1} Z_{1, \beta-1} \in U_{(2, \beta+1)} .
\end{aligned}
$$

It follows from the inductive hypothesis that $z_{1, \beta-1} \in R \subset U_{(2, \beta+1)}$. Thus we obtain

$$
\begin{aligned}
\sum_{l=l_{0}}^{-1} b_{l}^{\prime} Z_{2, \beta}^{l} & +\sum_{l=l_{0}}^{-1} q\left(q^{-2 l}-1\right) b_{l} Z_{1, \beta} Z_{2, \beta-1} Z_{2, \beta}^{l-1} \\
& -\left(z_{1, \beta}+z_{2, \beta-1}-z_{1, \beta-1}-z_{2, \beta}\right) Z_{1, \beta} Z_{2, \beta}^{-1} Z_{2, \beta-1} \in U_{(2, \beta+1)} .
\end{aligned}
$$

It follows from the inductive hypothesis and Proposition 2.3 (and Lemma 2.4 when $\beta=2)$ that $z_{1, \beta-1}, z_{1, \beta} b_{n-\beta+1}, z_{2, \beta-1}$ and $z_{2, \beta}$ belong to $R \subset U_{(2, \beta+1)}$. On the other hand, it follows from [4], Proposition 5.2.1, that $b_{n-\beta+1}=$ $Z_{1, \beta} Z_{2, \beta+1} \ldots Z_{n-\beta+1, n}$. Hence, $z_{1, \beta} Z_{1, \beta}$ belongs to $U_{(2, \beta+1)}$. Thus,

$$
\left(z_{1, \beta}+z_{2, \beta-1}-z_{1, \beta-1}-z_{2, \beta}\right) Z_{1, \beta} Z_{2, \beta-1} \in U_{(2, \beta+1)} .
$$

Multiplying (8) on the right by $Z_{2, \beta}$ leads to

$$
\sum_{l=l_{0}}^{-1} b_{l}^{\prime} Z_{2, \beta}^{l+1}+\sum_{l=l_{0}}^{-1} q\left(q^{-2 l}-1\right) b_{l} Z_{1, \beta} Z_{2, \beta-1} Z_{2, \beta}^{l} \in U_{(2, \beta+1)}
$$

In other words,

$$
\sum_{l=l_{0}+1}^{0} b_{l}^{\prime \prime} Z_{2, \beta}^{l}+q\left(q^{-2 l_{0}}-1\right) b_{l_{0}} Z_{1, \beta} Z_{2, \beta-1} Z_{2, \beta}^{l_{0}} \in U_{(2, \beta+1)} .
$$


As $U_{(2, \beta+1)}$ is a left $B$-module with basis $\left\{Z_{2, \beta}^{l}\right\}_{l \in \mathbb{N}}$, this implies that $b_{l_{0}}=0$, a contradiction. Hence $x_{-}=0$ and $x=x_{+} \in U_{(2, \beta+1)}$, as desired.

Step 2: we prove that $z_{1, \beta-1}+z_{2, \beta}=z_{1, \beta}+z_{2, \beta-1}$.

As $x_{-}=0$ and $z_{1, \beta-1} Z_{1, \beta-1} \in U_{(2, \beta+1)}$ by the inductive hypothesis, we deduce from (7) that

$$
y:=\left(z_{1, \beta}+z_{2, \beta-1}-z_{1, \beta-1}-z_{2, \beta}\right) Z_{1, \beta} Z_{2, \beta-1} \in U_{(2, \beta+1)} Z_{2, \beta} .
$$

Thus, $y$ is an element of $U_{(2, \beta+1)}$ which $q$-commutes with $Z_{1, \beta-1}$ and which belongs to $U_{(2, \beta+1)} Z_{2, \beta}$. As in the proof of Lemma 2.4 (Step 2), some easy calculations show that this forces $y=0$, so that

$$
z_{1, \beta-1}+z_{2, \beta}=z_{1, \beta}+z_{2, \beta-1},
$$

as desired.

Step 3: we prove that, for all $\alpha<\beta$, we have $z_{1, \alpha}+z_{2, \beta}=z_{1, \beta}+z_{2, \alpha}$.

First, when $\alpha=\beta-1$, the result follows from Step 2. Next, for $\alpha<\beta-1$, it follows from the inductive hypothesis that

$$
z_{1, \alpha}+z_{2, \beta-1}=z_{1, \beta-1}+z_{2, \alpha} .
$$

Further, it follows from Step 2 that

$$
z_{1, \beta-1}+z_{2, \beta}=z_{1, \beta}+z_{2, \beta-1} .
$$

Combining these two equalities leads to the desired result.

Step 4: we prove that $z_{1, \beta}$ belongs to $Z(R)$.

It follows from Proposition 2.3 that $z_{1, \beta}=Q_{1, \beta}\left(\Delta_{n}\right) \Delta_{n+1-\beta}^{-1}+P_{1, \beta}\left(\Delta_{n}\right)$, for some polynomials $Q_{1, \beta}\left(\Delta_{n}\right), P_{1, \beta}\left(\Delta_{n}\right) \in K\left[\Delta_{n}\right]$. Further, it follows from the inductive hypothesis and Proposition 2.3 (and Lemma 2.4 when $\beta=2$ ) that $z_{1, \beta-1}=P_{1, \beta-1}\left(\Delta_{n}\right), z_{2, \beta-1}=P_{2, \beta-1}\left(\Delta_{n}\right)$ and $z_{2, \beta}=P_{2, \beta}\left(\Delta_{n}\right)$, where each $P_{i, \alpha} \in K\left[\Delta_{n}\right]$. As $z_{1, \beta-1}+z_{2, \beta}=z_{1, \beta}+z_{2, \beta-1}$, we obtain

$$
P_{1, \beta-1}\left(\Delta_{n}\right)+P_{2, \beta}\left(\Delta_{n}\right)=Q_{1, \beta}\left(\Delta_{n}\right) \Delta_{n+1-\beta}^{-1}+P_{1, \beta}\left(\Delta_{n}\right)+P_{2, \beta-1}\left(\Delta_{n}\right) .
$$

Recalling that the monomials $\Delta_{1}^{i_{1}} \ldots \Delta_{n}^{i_{n}}$ with $i_{k} \in \mathbb{Z}$ are linearly independent, we get that

$$
Q_{1, \beta}\left(\Delta_{n}\right)=0,
$$

so that $z_{1, \beta}=P_{1, \beta}\left(\Delta_{n}\right)$ belongs to $K\left[\Delta_{n}\right]=Z(R)$.

Step 5: we prove that $D\left(Z_{i, \alpha}\right)=\operatorname{ad}_{x}\left(Z_{i, \alpha}\right)+z_{i, \alpha} Z_{i, \alpha}$ for all $(i, \alpha) \in \llbracket 1, n \rrbracket^{2}$. 
First, if $i \geq 2$ or $\alpha \geq \beta$, then $Z_{i, \alpha}=Y_{i, \alpha}^{(2, \beta)^{+}}=Y_{i, \alpha}^{(2, \beta)}$, so that the result easily follows from the inductive hypothesis.

Next, assume that $i=1$ and $\alpha<\beta$, so that $Z_{1, \alpha}=Y_{1, \alpha}^{(2, \beta+1)}=Y_{1, \alpha}^{(2, \beta)}+$ $Z_{1, \beta} Z_{2, \beta}^{-1} Z_{2, \alpha}$. Hence we deduce from the inductive hypothesis that

$$
\begin{aligned}
D\left(Z_{1, \alpha}\right)= & D\left(Y_{1, \alpha}^{(2, \beta)}+Z_{1, \beta} Z_{2, \beta}^{-1} Z_{2, \alpha}\right) \\
= & \operatorname{ad}_{x}\left(Y_{1, \alpha}^{(2, \beta)}\right)+z_{1, \alpha} Y_{1, \alpha}^{(2, \beta)} \\
& \quad+\operatorname{ad}_{x}\left(Z_{1, \beta} Z_{2, \beta}^{-1} Z_{2, \alpha}\right)+\left(z_{1, \beta}-z_{2, \beta}+z_{2, \alpha}\right) Z_{1, \beta} Z_{2, \beta}^{-1} Z_{2, \alpha} \\
= & \operatorname{ad}_{x}\left(Z_{1, \alpha}\right)+z_{1, \alpha} Z_{1, \alpha}+\left(z_{1, \beta}-z_{2, \beta}+z_{2, \alpha}-z_{1, \alpha}\right) Z_{1, \beta} Z_{2, \beta}^{-1} Z_{2, \alpha} .
\end{aligned}
$$

Now it follows from the Step 3 that $z_{1, \alpha}+z_{2, \beta}=z_{1, \beta}+z_{2, \alpha}$. Hence

$$
D\left(Z_{1, \alpha}\right)=\operatorname{ad}_{x}\left(Z_{1, \alpha}\right)+z_{1, \alpha} Z_{1, \alpha},
$$

as desired.

Lemma 2.6. Let $(j, \beta) \in E$ with $(j, \beta) \geq(3,1)$. Then:

1) $x \in U_{(j, \beta)}$.

2) For all $(k, \delta)<(j, \beta), i<k$ and $\alpha<\delta$, we have $z_{i, \alpha}+z_{k, \delta}=z_{i, \delta}+z_{k, \alpha}$.

3) $D\left(Y_{i, \alpha}^{(j, \beta)}\right)=\operatorname{ad}_{x}\left(Y_{i, \alpha}^{(j, \beta)}\right)+z_{i, \alpha} Y_{i, \alpha}^{(j, \beta)}$ for all $(i, \alpha) \in \llbracket 1, n \rrbracket^{2}$.

Proof. We prove this result by induction on $(j, \beta)$. The case $(j, \beta)=(3,1)$ follows from Lemma 2.5 .

Assume that the result is established for $(3,1) \leq(j, \beta)<(n, n+1)$, and let $(j, \beta)^{+}$be the smallest element of $E$ greater then $(j, \beta)$.

In order to simplify the notation, we set $Z_{i, \alpha}:=Y_{i, \alpha}^{(j, \beta)^{+}}$for all $(i, \alpha) \in \llbracket 1, n \rrbracket^{2}$. Moreover, for all $\gamma \in \mathcal{E}:=\mathbb{N}^{(j-1) n} \times\left(\mathbb{N}^{\beta-1} \times \mathbb{Z}^{n+1-\beta}\right) \times\left(\mathbb{N} \times \mathbb{Z}^{n-1}\right) \times \cdots$ $\times\left(\mathbb{N} \times \mathbb{Z}^{n-1}\right) \subset \mathbb{Z}^{n^{2}}$, set

$$
Z^{\underline{\gamma}}:=Z_{1,1}^{\gamma_{1,1}} Z_{1,2}^{\gamma_{1,2}} \ldots Z_{n, n}^{\gamma_{n, n}}
$$

We now proceed in four steps.

Step 1: we prove that $x \in U_{(j, \beta)^{+}}$.

It follows from the inductive hypothesis that $x$ belongs to $U_{(j, \beta)}$. We distinguish between two cases.

If $\beta=1$, then $U_{(j, \beta)^{+}}=U_{(j, \beta)}$, so that the induction step is obvious in this case.

Now, assume that $\beta>1$. In this case, using the notation of the previous section,

$$
U_{(j, \beta)}=U_{(j, \beta)}+\Sigma_{j, \beta}^{-1},
$$


so that $x$ can be written as

$$
x=\sum_{\underline{\gamma} \in \mathcal{E}} c_{\underline{\gamma}} Z^{\underline{\gamma}}
$$

with $c_{\underline{\gamma}} \in K$. Set

$$
x_{+}:=\sum_{\substack{\underline{\underline{\gamma}} \in \mathcal{E} \\ \gamma_{j, \beta} \geq 0}} c_{\underline{\gamma}} Z^{\underline{\gamma}}, \quad x_{-}:=\sum_{\substack{\underline{\gamma} \in \mathcal{E} \\ \gamma_{j, \beta}<0}} c_{\underline{\gamma}} Z^{\underline{\gamma}} .
$$

Assume that $x_{-} \neq 0$.

Denote by $B$ the subalgebra of $U_{(j, \beta)}=U_{(j, \beta)^{+}} \Sigma_{j, \beta}^{-1}$ generated by the $Z_{i, \alpha}$ with $(i, \alpha) \neq(j, \beta)$ and the $Z_{i, \alpha}^{-1}$ such that $i \geq 2$ and $\alpha \geq 2$ but $(i, \alpha)>(j, \beta)$. Then $U_{(j, \beta)}=U_{(j, \beta)}+\Sigma_{j, \beta}^{-1}$ is a left $B$-module with basis $\left\{Z_{j, \beta}^{l}\right\}_{l \in \mathbb{Z}}$, so that there are elements $b_{l} \in B$ such that

$$
x_{-}=\sum_{l=l_{0}}^{-1} b_{l} Z_{j, \beta}^{l}
$$

with $l_{0}<0$ and $b_{l_{0}} \neq 0$. (Observe that this makes sense because we have assumed that $x_{-} \neq 0$.)

The derivation $D$ of $R$ extends to a derivation of $U_{(j, \beta)^{+}}$, since $U_{(j, \beta)^{+}}$is obtained from $R$ by a sequence of localisations; so $D\left(Z_{j-1, \beta-1}\right) \in U_{(j, \beta)^{+}}$. This implies that

$$
\begin{aligned}
& x_{-} Z_{j-1, \beta-1}-Z_{j-1, \beta-1} x_{-}+z_{j-1, \beta-1} Z_{j-1, \beta-1} \\
& \quad+\left(z_{j-1, \beta}+z_{j, \beta-1}-z_{j-1, \beta-1}-z_{j, \beta}\right) Z_{j-1, \beta} Z_{j, \beta}^{-1} Z_{j, \beta-1} \in U_{(j, \beta)^{+}} .
\end{aligned}
$$

Now

$$
Z_{j, \beta}^{-k} Z_{j-1, \beta-1}=Z_{j-1, \beta-1} Z_{j, \beta}^{-k}+q\left(q^{2 k}-1\right) Z_{j-1, \beta} Z_{j, \beta-1} Z_{j, \beta}^{-k-1}
$$

for all positive integers $k$. Hence

$$
\begin{aligned}
x_{-} Z_{j-1, \beta-1}- & Z_{j-1, \beta-1} x_{-}+z_{j-1, \beta-1} Z_{j-1, \beta-1} \\
& +\left(z_{j-1, \beta}+z_{j, \beta-1}-z_{j-1, \beta-1}-z_{j, \beta}\right) Z_{j-1, \beta} Z_{j, \beta}^{-1} Z_{j, \beta-1} \\
= & \sum_{l=l_{0}}^{-1} b_{l}^{\prime} Z_{j, \beta}^{l}+\sum_{l=l_{0}}^{-1} q\left(q^{-2 l}-1\right) b_{l} Z_{j-1, \beta} Z_{j, \beta-1} Z_{j, \beta}^{l-1} \\
& \quad-\left(z_{j-1, \beta}+z_{j, \beta-1}-z_{j-1, \beta-1}-z_{j, \beta}\right) Z_{j-1, \beta} Z_{j, \beta}^{-1} Z_{j, \beta-1} \\
& +z_{j-1, \beta-1} Z_{j-1, \beta-1} \in U_{(j, \beta)^{+}} .
\end{aligned}
$$

Now observe that $z_{j-1, \beta-1} \in R \subset U_{(j, \beta)^{+}}$. Indeed, if $\beta>2$, then it follows from Proposition 2.3 that $z_{j-1, \beta-1}$ also belongs to $R \subset U_{(j, \beta)^{+}}$. On the other hand, if 
$\beta=2$, then it follows from the inductive hypothesis that $z_{j-1,1}+z_{1,2}=z_{1,1}+$ $z_{j-1,2}$. As each of $z_{1,1}, z_{1,2}$ and $z_{j-1,2}$ belong to $R \subset U_{(j, \beta)}+$ by Lemma 2.4 and Proposition 2.3, it follows that $z_{j-1,1} \in R \subset U_{(j, \beta)^{+}}$.

As $z_{j-1, \beta-1} \in R \subset U_{(j, \beta)^{+}}$, we obtain

$$
\begin{aligned}
\sum_{l=l_{0}}^{-1} b_{l}^{\prime} Z_{j, \beta}^{l}+\sum_{l=l_{0}}^{-1} q\left(q^{-2 l}-1\right) b_{l} Z_{j-1, \beta} Z_{j, \beta-1} Z_{j, \beta}^{l-1} \\
\quad-\left(z_{j-1, \beta}+z_{j, \beta-1}-z_{j-1, \beta-1}-z_{j, \beta}\right) Z_{j-1, \beta} Z_{j, \beta}^{-1} Z_{j, \beta-1} \in U_{(j, \beta)^{+}} .
\end{aligned}
$$

It follows from Proposition 2.3 that $z_{j-1, \beta}$ and $z_{j, \beta}$ belong to $R \subset U_{(j, \beta)^{+}}$; so each of $z_{j-1, \beta-1}, z_{j-1, \beta}$ and $z_{j, \beta}$ also belong to $U_{(j, \beta)^{+}}$.

We now distinguish between two cases to prove that

$$
\left(z_{j-1, \beta}+z_{j, \beta-1}-z_{j-1, \beta-1}-z_{j, \beta}\right) Z_{j-1, \beta} Z_{j, \beta-1} \in U_{(j, \beta)^{+}} .
$$

(Note that it only remains to show that $z_{j, \beta-1} Z_{j-1, \beta} Z_{j, \beta-1} \in U_{(j, \beta)^{+}}$.)

First, if $\beta=2$, then it follows from Proposition 2.3 that $z_{j, \beta-1} b_{n+j-1} \in R \subset$ $U_{(j, \beta)^{+}}$. On the other hand, it follows from [4], Proposition 5.2.1, that $b_{n+j-1}=$ $Z_{j, 1} Z_{j+1,2} \ldots Z_{n, n-j+1}$. Hence $z_{j, \beta-1} Z_{j, \beta-1}$ belongs to $U_{(j, \beta)}+$ since $Z_{j+1,2}, \ldots$, $Z_{n, n-j+1}$ are invertible in $U_{(j, \beta)^{+}}$. Thus,

$$
\left(z_{j-1, \beta}+z_{j, \beta-1}-z_{j-1, \beta-1}-z_{j, \beta}\right) Z_{j-1, \beta} Z_{j, \beta-1} \in U_{(j, \beta)^{+}},
$$

as claimed.

If $\beta>2$, then $\beta-1 \geq 2$, and so it follows from Proposition 2.3 that $z_{j, \beta-1} \in$ $R \subset U_{(j, \beta)^{+}}$. Thus,

$$
\left(z_{j-1, \beta}+z_{j, \beta-1}-z_{j-1, \beta-1}-z_{j, \beta}\right) Z_{j-1, \beta} Z_{j, \beta-1} \in U_{(j, \beta)^{+}},
$$

as claimed.

So, in each case, $\left(z_{j-1, \beta}+z_{j, \beta-1}-z_{j-1, \beta-1}-z_{j, \beta}\right) Z_{j-1, \beta} Z_{j, \beta-1} \in U_{(j, \beta)^{+}}$, and thus multiplying (10) on the right by $Z_{j, \beta}$ leads to

$$
\sum_{l=l_{0}}^{-1} b_{l}^{\prime} Z_{j, \beta}^{l+1}+\sum_{l=l_{0}}^{-1} q\left(q^{-2 l}-1\right) b_{l} Z_{j-1, \beta} Z_{j, \beta-1} Z_{j, \beta}^{l} \in U_{(j, \beta)^{+}}
$$

In other words, we have

$$
\sum_{l=l_{0}+1}^{0} b_{l}^{\prime \prime} Z_{j, \beta}^{l}+q\left(q^{-2 l_{0}}-1\right) b_{l_{0}} Z_{j-1, \beta} Z_{j, \beta-1} Z_{j, \beta}^{l_{0}} \in U_{(j, \beta)^{+}} .
$$

As $U_{(j, \beta)^{+}}$is a left $B$-module with basis $\left\{Z_{j, \beta}^{l}\right\}_{l \in \mathbb{N}}$, this implies that $b_{l_{0}}=0$, a contradiction. Hence $x_{-}=0$ and $x=x_{+} \in U_{(j, \beta)^{+}}$, as desired. 
Step 2: we prove that $z_{j-1, \beta-1}+z_{j, \beta}=z_{j-1, \beta}+z_{j, \beta-1}$.

As $x_{-}=0$ and $z_{j-1, \beta-1} Z_{j-1, \beta-1} \in U_{(j, \beta)^{+}}$by the above study, we deduce from (9) that

$$
y:=\left(z_{j-1, \beta}+z_{j, \beta-1}-z_{j-1, \beta-1}-z_{j, \beta}\right) Z_{j-1, \beta} Z_{j, \beta-1} \in U_{(j, \beta)^{+}} Z_{j, \beta} .
$$

Thus, $y$ is an element of $U_{(j, \beta)}+$ which $q$-commutes with $Z_{j-1, \beta-1}$ and which belongs to $U_{(j, \beta)}+Z_{j, \beta}$. As in the proof of Lemma 2.4 (Step 2), some easy calculations show that this forces $y=0$, so that

$$
z_{j-1, \beta-1}+z_{j, \beta}=z_{j-1, \beta}+z_{j, \beta-1},
$$

as desired.

Step 3: we prove that $z_{i, \alpha}+z_{k, \delta}=z_{i, \delta}+z_{k, \alpha}$, for all $(k, \delta)<(j, \beta)^{+}$, with $i<k$ and $\alpha<\delta$.

In order to do this, let $(k, \delta)<(j, \beta)^{+}$, with $i<k$ and $\alpha<\delta$. If $(k, \delta)<(j, \beta)$, it follows from the inductive hypothesis that $z_{i, \alpha}+z_{k, \delta}=z_{i, \delta}+z_{k, \alpha}$, as required. Now we assume that $(k, \delta)=(j, \beta)$.

First, if $(i, \alpha)=(j-1, \beta-1)$, then we have just proved in Step 2 that $z_{i, \alpha}+z_{j, \beta}=$ $z_{i, \beta}+z_{j, \alpha}$, as required.

Next, assume that $i<j-1$ and $\alpha=\beta-1$. Then it follows from the inductive hypothesis that

$$
z_{i, \beta-1}+z_{j-1, \beta}=z_{i, \beta}+z_{j-1, \beta-1} .
$$

Moreover, we have already shown that $z_{j-1, \beta}+z_{j, \beta-1}=z_{j-1, \beta-1}+z_{j, \beta}$. Hence,

$$
z_{i, \beta-1}+z_{j, \beta}=z_{i, \beta}+z_{j, \beta-1},
$$

as required. Similar arguments show that

$$
z_{j-1, \alpha}+z_{j, \beta}=z_{j-1, \beta}+z_{j, \alpha}
$$

for all $\alpha<\beta$.

Assume now that $i<j-1$ and $\alpha<\beta-1$. It follows from the inductive hypothesis that

$$
z_{i, \alpha}+z_{j-1, \beta}=z_{i, \beta}+z_{j-1, \alpha} .
$$

Moreover, we have already shown that

$$
z_{j-1, \alpha}+z_{j, \beta}=z_{j-1, \beta}+z_{j, \alpha} .
$$

Combining these two equations leads to

$$
z_{i, \alpha}+z_{j, \beta}=z_{i, \beta}+z_{j, \alpha},
$$

as desired. 
Step 4: we prove that $D\left(Z_{i, \alpha}\right)=\operatorname{ad}_{x}\left(Z_{i, \alpha}\right)+z_{i, \alpha} Z_{i, \alpha}$ for all $(i, \alpha) \in \llbracket 1, n \rrbracket^{2}$.

First, if $i \geq j$ or $\alpha \geq \beta$, then $Z_{i, \alpha}=Y_{i, \alpha}^{(j, \beta)^{+}}=Y_{i, \alpha}^{(j, \beta)}$; so that the result easily follows from the inductive hypothesis.

Now assume that $i<j$ and $\alpha<\beta$, so that $Z_{i, \alpha}=Y_{i, \alpha}^{(j, \beta)^{+}}=Y_{i, \alpha}^{(j, \beta)}+$ $Z_{i, \beta} Z_{j, \beta}^{-1} Z_{j, \alpha}$. Hence, we deduce from the inductive hypothesis (and the previous case) that

$$
\begin{aligned}
D\left(Z_{i, \alpha}\right)= & D\left(Y_{i, \alpha}^{(j, \beta)}+Z_{i, \beta} Z_{j, \beta}^{-1} Z_{j, \alpha}\right) \\
= & \operatorname{ad}_{x}\left(Y_{i, \alpha}^{(j, \beta)}\right)+z_{i, \alpha} Y_{i, \alpha}^{(j, \beta)} \\
& \quad+\operatorname{ad}_{x}\left(Z_{i, \beta} Z_{j, \beta}^{-1} Z_{j, \alpha}\right)+\left(z_{i, \beta}-z_{j, \beta}+z_{j, \alpha}\right) Z_{i, \beta} Z_{j, \beta}^{-1} Z_{j, \alpha} \\
= & \operatorname{ad}_{x}\left(Z_{i, \alpha}\right)+z_{i, \alpha} Z_{i, \alpha}+\left(z_{i, \beta}-z_{j, \beta}+z_{j, \alpha}-z_{i, \alpha}\right) Z_{i, \beta} Z_{j, \beta}^{-1} Z_{j, \alpha}
\end{aligned}
$$

Now it follows from Step 3 that $z_{i, \beta}-z_{j, \beta}+z_{j, \alpha}-z_{i, \alpha}=0$. Hence

$$
D\left(Z_{i, \alpha}\right)=\operatorname{ad}_{x}\left(Z_{i, \alpha}\right)+z_{i, \alpha} Z_{i, \alpha}
$$

as desired.

Corollary 2.7. The element $z_{i, \alpha}$ belongs to $Z(R)=K\left[\Delta_{n}\right]$ for all $(i, \alpha) \in \llbracket 1, n \rrbracket^{2}$.

Proof. We already know from Proposition 2.3 that $z_{i, \alpha} \in Z(R)$ when $i \geq 2$ and $\alpha \geq 2$. Further, it follows from Lemma 2.5 that $z_{i, \alpha} \in Z(R)$ when $i=1$. Finally, let $i \geq 2$. It follows from Lemma 2.6 that $z_{i, 1}=z_{1,1}+z_{i, 2}-z_{1,2}$. Thus, $z_{i, 1} \in Z(R)$, since the three elements on the right side of this equation belong to $Z(R)$.

Corollary 2.8. Any derivation $D$ of $R=\mathcal{O}_{q}\left(M_{n}\right)=K\left[Y_{i, \alpha}\right]$ can be written as $D=\mathrm{a} d_{x}+\theta$, where $x \in R$ and $\theta$ is a derivation of $R$ such that $\theta\left(Y_{i, \alpha}\right)=z_{i, \alpha} Y_{i, \alpha}$ for some $z_{i, \alpha} \in K[\Delta]$ satisfying $z_{i, \alpha}+z_{k, \delta}=z_{i, \delta}+z_{k, \alpha}$ whenever $i<k$ and $\alpha<\delta$.

Proof. This is the case $(n, n+1)$ of Lemma 2.6.

We now seek to describe the possibilities for the derivation $\theta$ occurring in the previous result.

It is easy to verify that there are $2 n$ derivations of $R$ given by $D_{i, *}, D_{*, \alpha}$, for $1 \leq i, \alpha \leq n$, where

$$
D_{i, *}\left(Y_{j, \beta}\right)=\delta_{i j} Y_{i, \beta} \quad \text { and } \quad D_{*, \alpha}\left(Y_{j, \beta}\right)=\delta_{\alpha \beta} Y_{j, \alpha} .
$$

In other words, $D_{i, *}$ fixes row $i$ and kills all the other rows, while $D_{*, \alpha}$ fixes column $\alpha$ and kills all other columns.

We show that $\theta$ above can be described in terms of these row and column derivations. However, note that these derivations are not independent, since $\sum D_{i, *}=$ 
$\sum D_{*, \alpha}$; so we begin by defining $2 n-1$ derivations which span the same space, but which are independent.

Set

$$
D_{j}= \begin{cases}D_{*, n+1-j} & \text { for } 1 \leq j \leq n-1, \\ D_{j-n+1, *} & \text { for } n+1 \leq j \leq 2 n-1,\end{cases}
$$

while

$$
D_{n}=D_{1, *}+D_{*, 1}-\sum_{i=1}^{n} D_{i, *} \quad\left(=D_{1, *}+D_{*, 1}-\sum_{\alpha=1}^{n} D_{*, \alpha}\right) .
$$

It is easy to see that the $K$-span of $\left\{D_{j} \mid 1 \leq j \leq 2 n-1\right\}$ is the same as the $K$-span of $\left\{D_{i, *}, D_{*, \alpha} \mid 1 \leq i, \alpha \leq n\right\}$.

Note that:

- If $j \in \llbracket 1, n-1 \rrbracket$, then $D_{j}\left(Y_{i, \alpha}\right)=Y_{i, \alpha}$ if $\alpha=n+1-j$, and $D_{j}\left(Y_{i, \alpha}\right)=0$ otherwise.

- $D_{n}\left(Y_{1,1}\right)=Y_{1,1}, D_{n}\left(Y_{i, \alpha}\right)=-Y_{i, \alpha}$ if $i \geq 2$ and $\alpha \geq 2$, and $D_{n}\left(Y_{i, \alpha}\right)=0$ otherwise.

- If $j \in \llbracket n+1,2 n-1 \rrbracket$, then $D_{j}\left(Y_{i, \alpha}\right)=Y_{i, \alpha}$ if $i=j-n+1$, and $D_{j}\left(Y_{i, \alpha}\right)=0$ otherwise.

It follows from Corollary 2.8 that any derivation $D$ of $R$ can be written as follows:

$$
D=\operatorname{ad}_{x}+z_{1, n} D_{1}+\cdots+z_{1,2} D_{n-1}+z_{1,1} D_{n}+z_{2,1} D_{n+1} \cdots+z_{n, 1} D_{2 n-1},
$$

with $x \in R$ and $z_{1,1}, \ldots, z_{1, n}, z_{2,1}, \ldots, z_{n, 1} \in Z(R)$.

Recall that the Hochschild cohomology group in degree 1 of $R$, denoted by $\mathrm{HH}^{1}(R)$, is defined by

$$
\operatorname{HH}^{1}(R):=\operatorname{Der}(R) / \operatorname{InnDer}(R),
$$

where $\operatorname{InnDer}(R):=\left\{\operatorname{ad}_{x} \mid x \in R\right\}$ is the Lie algebra of inner derivations of $R$. Note that $\mathrm{HH}^{1}(R)$ is sometimes denoted by $\mathrm{HH}^{1}(R, R)$ in the literature. It is well known that $\mathrm{HH}^{1}(R)$ is a module over $\mathrm{HH}^{0}(R):=Z(R)$. The following result makes this latter structure precise.

Theorem 2.9. 1) Every derivation $D$ of $R$ can be uniquely written as

$$
D=\operatorname{ad}_{x}+\mu_{1} D_{1}+\cdots+\mu_{2 n-1} D_{2 n-1},
$$

with $\operatorname{ad}_{x} \in \operatorname{InnDer}(R)$ and $\mu_{1}, \ldots, \mu_{2 n-1} \in Z(R)=K\left[\Delta_{n}\right]$.

2) $\mathrm{HH}^{1}(R)$ is a free $Z(R)$-module of rank $2 n-1$ with basis $\left(\overline{D_{1}}, \ldots, \overline{D_{2 n-1}}\right)$. 
Proof. It just remains to prove that if $x \in R$ and $\mu_{1}, \ldots, \mu_{2 n-1} \in Z(R)$ with $\operatorname{ad}_{x}+\mu_{1} D_{1}+\cdots+\mu_{2 n-1} D_{2 n-1}=0$, then $\mu_{1}=\cdots=\mu_{2 n-1}=0$ and $\operatorname{ad}_{x}=0$. Set $\theta:=\mu_{1} D_{1}+\cdots+\mu_{2 n-1} D_{2 n-1}$, so that $\operatorname{ad}_{x}+\theta=0$. The derivation $\theta$ of $R$ extends uniquely to a derivation $\tilde{\theta}$ of the quantum torus $P(\Lambda)$. Naturally, we still have $\operatorname{ad}_{x}+\tilde{\theta}=0$. Further, straightforward computations show that

$$
\tilde{\theta}\left(T_{i, \alpha}\right)= \begin{cases}\mu_{n} T_{1,1} & \text { if } \alpha \geq 2, \\ \mu_{n+1-\alpha} T_{1, \alpha} & \text { if } \mathrm{i} \geq 2, \\ \mu_{n+i-1} T_{i, 1} & \text { otherwise. }\end{cases}
$$

Hence $\tilde{\theta}$ is a central derivation of $P(\Lambda)$, in the terminology of [12]. Thus we deduce from [12], Corollary 2.3, that $\operatorname{ad}_{x}=0=\theta$. Evaluating $\theta$ on $Y_{1, \alpha}$ with $\alpha \in \llbracket 1, n \rrbracket$, and on $Y_{i, 1}$ with $i \in \llbracket 1, n \rrbracket$ leads to $\mu_{1}=\cdots=\mu_{2 n-1}=0$, as desired.

As a corollary of Theorem 2.9, we obtain some new information on the twisted homology of quantum matrices. We refer to [7] and references therein for definition and properties of the twisted homology. In [7], the authors have shown using results of [14] that the "dimension drop" in Hochschild homology is overcome by passing to twisted Hochschild homology. More precisely, they have shown that

$$
\mathrm{HH}_{n^{2}}\left(\mathcal{O}_{q}\left(M_{n}\right), \mathcal{O}_{q}\left(M_{n}\right)_{\sigma}\right) \simeq K\left[\Delta_{n}\right],
$$

where $\sigma$ denotes the automorphism of $\mathcal{O}_{q}\left(M_{n}\right)$ defined by

$$
\sigma\left(Y_{i, \alpha}\right)=q^{2(n+1-i-\alpha)} Y_{i, \alpha},
$$

for all $(i, \alpha) \in \llbracket 1, n \rrbracket$. In fact, it is a consequence of Theorem 2.9 and [7], Proposition 2.1, that not only $\operatorname{HH}_{n^{2}}\left(\mathcal{O}_{q}\left(M_{n}\right), \mathcal{O}_{q}\left(M_{n}\right)_{\sigma}\right)$ is nonzero, but also $\mathrm{HH}_{n^{2}-1}\left(\mathcal{O}_{q}\left(M_{n}\right), \mathcal{O}_{q}\left(M_{n}\right)_{\sigma}\right)$ is nonzero. More precisely, recall from [7], Proposition 2.1, that $\mathcal{O}_{q}\left(M_{n}\right)$ has the (twisted) Poincaré duality property, so that $\mathrm{HH}_{n^{2}-1}\left(\mathcal{O}_{q}\left(M_{n}\right), \mathcal{O}_{q}\left(M_{n}\right)_{\sigma}\right)$ is isomorphic as a vector space to $\operatorname{HH}^{1}\left(\mathcal{O}_{q}\left(M_{n}\right)\right)$. Hence we deduce from Theorem 2.9 the following result.

Corollary 2.10. $\mathrm{HH}_{n^{2}-1}\left(\mathcal{O}_{q}\left(M_{n}\right), \mathcal{O}_{q}\left(M_{n}\right)_{\sigma}\right) \neq 0$.

\section{On Hochschild cohomology and twisted Hochschild homology of $\mathcal{O}_{q}\left(\mathrm{GL}_{n}\right)$ and $\mathcal{O}_{q}\left(\mathrm{SL}_{n}\right)$}

In this section, we describe the derivations of $\mathcal{O}_{q}\left(\mathrm{GL}_{n}\right)$ and $\mathcal{O}_{q}\left(\mathrm{SL}_{n}\right)$. As a consequence, we show that the Hochschild cohomology group in degree 1 and the twisted Hochschild homology group in degree $n^{2}-2$ of $\mathcal{O}_{q}\left(\mathrm{SL}_{n}\right)$ are both finite-dimensional vector spaces of dimension $2 n-2$. 
3.1. Derivations of $\boldsymbol{O}_{q}\left(\mathbf{G L}_{n}\right)$. The quantisation of the ring of regular functions on $\mathrm{GL}_{n}(K)$ is denoted by $\mathcal{O}_{q}\left(\mathrm{GL}_{n}\right)$; recall that it is the localisation of $\mathcal{O}_{q}\left(M_{n}\right)$ at the powers of the central element $\Delta_{n}$. It is well-known that $\mathcal{O}_{q}\left(\mathrm{GL}_{n}\right)$ is a Noetherian domain that is endowed with a Hopf algebra structure.

As $\mathcal{O}_{q}\left(\mathrm{GL}_{n}\right)$ is a localisation of $\mathcal{O}_{q}\left(M_{n}\right)$, the derivations $D_{1}, \ldots, D_{2 n-1}$ of $\mathcal{O}_{q}\left(M_{n}\right)$ defined in the discussion before Theorem 2.9 extend uniquely to derivations of $\mathcal{O}_{q}\left(\mathrm{GL}_{n}\right)$ that are still denoted by $D_{1}, \ldots, D_{2 n-1}$.

Theorem 3.1. 1) Every derivation $D$ of $\mathcal{O}_{q}\left(\mathrm{GL}_{n}\right)$ can be uniquely written as

$$
D=\operatorname{ad}_{x}+\mu_{1} D_{1}+\cdots+\mu_{2 n-1} D_{2 n-1},
$$

with $\operatorname{ad}_{x} \in \operatorname{InnDer}\left(\mathcal{O}_{q}\left(\mathrm{GL}_{n}\right)\right)$ and $\mu_{1}, \ldots, \mu_{2 n-1} \in Z\left(\mathcal{O}_{q}\left(\mathrm{GL}_{n}\right)\right)=K\left[\Delta_{n}^{ \pm 1}\right]$.

2) $\mathrm{HH}^{1}\left(\mathcal{O}_{q}\left(\mathrm{GL}_{n}\right)\right)$ is a free $Z\left(\mathcal{O}_{q}\left(\mathrm{GL}_{n}\right)\right)$-module of rank $2 n-1$ with basis $\left(\bar{D}_{1}, \ldots, \overline{D_{2 n-1}}\right)$.

Proof. Let $D$ be a derivation of $\mathcal{O}_{q}\left(\mathrm{GL}_{n}\right)$. Then there exists $k \in \mathbb{N}$ such that, for all $(i, \alpha) \in \llbracket 1, n \rrbracket^{2}$,

$$
\Delta_{n}^{k} D\left(Y_{i, \alpha}\right)=D\left(Y_{i, \alpha}\right) \Delta_{n}^{k} \in \mathcal{O}_{q}\left(M_{n}\right) .
$$

It is easy to check that $\Delta_{n}^{k} . D$ restricts to a derivation of $\mathcal{O}_{q}\left(M_{n}\right)$. Hence it follows from Theorem 2.9 that there exist $\mu_{1}, \ldots, \mu_{2 n-1} \in K\left[\Delta_{n}\right]$ and $x \in \mathcal{O}_{q}\left(M_{n}\right)$ such that

$$
\Delta_{n}^{k} . D=\operatorname{ad}_{x}+\mu_{1} D_{1}+\cdots+\mu_{2 n-1} D_{2 n-1} .
$$

As $\Delta_{n}$ is central, we obtain

$$
D=\operatorname{ad}_{\Delta_{n}^{-k} x}+\mu_{1} \Delta_{n}^{-k} D_{1}+\cdots+\mu_{2 n-1} \Delta_{n}^{-k} D_{2 n-1},
$$

as desired.

It just remains to prove that if $x \in \mathcal{O}_{q}\left(\mathrm{GL}_{n}\right)$ and $\mu_{1}, \ldots, \mu_{2 n-1} \in Z\left(\mathcal{O}_{q}\left(\mathrm{GL}_{n}\right)\right)$ with $\operatorname{ad}_{x}+\mu_{1} D_{1}+\cdots+\mu_{2 n-1} D_{2 n-1}=0$, then $\mu_{1}=\cdots=\mu_{2 n-1}=0$ and $\operatorname{ad}_{x}=0$. Set $D:=\operatorname{ad}_{x}+\mu_{1} D_{1}+\cdots+\mu_{2 n-1} D_{2 n-1}$. Let $k \in \mathbb{N}$ such that $x \Delta_{n}^{k} \in \mathcal{O}_{q}\left(M_{n}\right)$ and $\mu_{i} \Delta_{n}^{k} \in \mathcal{O}_{q}\left(M_{n}\right)$ for all $i \in \llbracket 1,2 n-1 \rrbracket$. Then $\Delta_{n}^{k} D$ induces a derivation of $\mathcal{O}_{q}\left(M_{n}\right)$ such that $0=\Delta_{n}^{k} D=\operatorname{ad}_{x \Delta_{n}^{k}}+\mu_{1} \Delta_{n}^{k} D_{1}+\cdots$ $+\mu_{2 n-1} \Delta_{n}^{k} D_{2 n-1}$. As all the $\mu_{i} \Delta_{n}^{k}$ belong to $K\left[\Delta_{n}\right]=Z\left(\mathcal{O}_{q}\left(M_{n}\right)\right)$, we deduce from Theorem 2.9 that $\Delta_{n}^{k} \cdot \operatorname{ad}_{x}=\operatorname{ad}_{\Delta_{n}^{k} x}=0$ and $\mu_{i} \Delta_{n}^{k}=0$ for all $i \in \llbracket 1,2 n-1 \rrbracket$. Naturally, this forces $\operatorname{ad}_{x}=0$ and $\mu_{i} \stackrel{2}{=} 0$ for all $i \in \llbracket 1,2 n-1 \rrbracket$, as required.

Following the same reasoning as in the discussion before Corollary 2.10, we obtain the following result regarding the twisted Hochschild homology of $\mathcal{O}_{q}\left(\mathrm{GL}_{n}\right)$.

Corollary 3.2. $\mathrm{HH}_{n^{2}-1}\left(\mathcal{O}_{q}\left(\mathrm{GL}_{n}\right), \mathcal{O}_{q}\left(\mathrm{GL}_{n}\right)_{\sigma}\right) \neq 0$. 
3.2. Derivations of $\boldsymbol{O}_{\boldsymbol{q}}\left(\mathbf{S L}_{\boldsymbol{n}}\right)$. In this section, we first consider the case where $n \geq 3$. (The case $n=2$ needs a slightly different treatment for technical reasons.)

The quantisation of the ring of regular functions on $\mathrm{SL}_{n}(K)$ is denoted by $\mathcal{O}_{q}\left(\mathrm{SL}_{n}\right)$; recall that

$$
\mathcal{O}_{q}\left(\mathrm{SL}_{n}\right):=\mathcal{O}_{q}\left(M_{n}\right) /\left\langle\Delta_{n}-1\right\rangle .
$$

We set $X_{i, \alpha}:=Y_{i, \alpha}+\left\langle\Delta_{n}-1\right\rangle$ for all $(i, \alpha) \in \llbracket 1, n \rrbracket^{2}$. It is well-known that $\mathcal{O}_{q}\left(\mathrm{SL}_{n}\right)$ is a Noetherian domain whose centre is reduced to scalars.

Observe that, for all $i \in \llbracket 1, n-1 \rrbracket \cup \llbracket n+1,2 n-1 \rrbracket$, the derivation $D_{i}+\frac{1}{n-2} D_{n}$ of $\mathcal{O}_{q}\left(M_{n}\right)$ satisfies $\left(D_{i}+\frac{1}{n-2} D_{n}\right)\left(\Delta_{n}\right)=0$. Hence it induces a derivation of $\mathcal{O}_{q}\left(\mathrm{SL}_{n}\right)$ that we denote by $D_{i}^{\prime}$.

Theorem 3.3. 1) Every derivation $D^{\prime}$ of $\mathcal{O}_{q}\left(\mathrm{SL}_{n}\right)$ can be uniquely written as follows:

$$
D^{\prime}=\operatorname{ad}_{y}+\mu_{1}^{\prime} D_{1}^{\prime}+\cdots+\mu_{n-1}^{\prime} D_{n-1}^{\prime}+\mu_{n+1}^{\prime} D_{n+1}^{\prime}+\cdots+\mu_{2 n-1}^{\prime} D_{2 n-1}^{\prime},
$$

$\operatorname{ad}_{y} \in \operatorname{InnDer}\left(\mathcal{O}_{q}\left(\mathrm{SL}_{n}\right)\right)$ and $\mu_{1}^{\prime}, \ldots, \mu_{n-1}^{\prime}, \mu_{n+1}^{\prime}, \ldots, \mu_{2 n-1}^{\prime} \in Z\left(\mathcal{O}_{q}\left(\mathrm{SL}_{n}\right)\right)=K$.

2) $\mathrm{HH}^{1}\left(\mathcal{O}_{q}\left(\mathrm{SL}_{n}\right)\right)$ is a finite-dimensional vector space of dimension $2 n-2$ with basis $\left(\overline{D_{1}^{\prime}}, \ldots, \overline{D_{n-1}^{\prime}}, \overline{D_{n+1}^{\prime}}, \ldots, \overline{D_{2 n-1}^{\prime}}\right)$.

Proof. Let $D^{\prime}$ be a derivation of $\mathcal{O}_{q}\left(\mathrm{SL}_{n}\right)$. Naturally, one can extend $D^{\prime}$ to a derivation of $\mathcal{O}_{q}\left(\mathrm{SL}_{n}\right)\left[t^{ \pm 1}\right]$ by setting $D^{\prime}(t)=0$. Now, recall from [11, Proposition] that there exists a unique isomorphism $\varphi: \mathcal{O}_{q}\left(\mathrm{SL}_{n}\right)\left[t^{ \pm 1}\right] \rightarrow \mathcal{O}_{q}\left(\mathrm{GL}_{n}\right)$ such that $\varphi\left(X_{i, \alpha}\right)=Y_{i, \alpha}$ if $i>1, \varphi\left(X_{1, \alpha}\right)=Y_{1, \alpha} \Delta_{n}^{-1}$, and $\varphi(t)=\Delta_{n}$. As $D^{\prime}$ is a derivation of $\mathcal{O}_{q}\left(\mathrm{SL}_{n}\right)\left[t^{ \pm 1}\right]$, one can transfer it via $\varphi$ in order to obtain a derivation of $\mathcal{O}_{q}\left(\mathrm{GL}_{n}\right)$. More precisely, it is easy to check that $D:=\varphi \circ D^{\prime} \circ \varphi^{-1}$ is a derivation of $\mathcal{O}_{q}\left(\mathrm{GL}_{n}\right)$ such that $D\left(\Delta_{n}\right)=0$. Hence, it follows from the proof of Theorem 3.1 that there exist $k \in \mathbb{N}, \mu_{1}, \ldots, \mu_{2 n-1} \in K\left[\Delta_{n}\right]$ and $x \in \mathcal{O}_{q}\left(M_{n}\right)$ such that $D=\Delta_{n}^{-k} \operatorname{ad}_{x}+$ $\Delta_{n}^{-k} \mu_{1} D_{1}+\cdots+\Delta_{n}^{-k} \mu_{2 n-1} D_{2 n-1}$. Moreover, since $D\left(\Delta_{n}\right)=0$, we must have $\mu_{1}+\cdots+\mu_{n-1}+\mu_{n+1}+\cdots+\mu_{2 n-1}-(n-2) \mu_{n}=0$. Hence $D=\Delta_{n}^{-k} \operatorname{ad}_{x}+$ $\Delta_{n}^{-k} \mu_{1} D_{1}^{\prime \prime}+\cdots+\Delta_{n}^{-k} \mu_{n-1} D_{n-1}^{\prime \prime}+\Delta_{n}^{-k} \mu_{n+1} D_{n+1}^{\prime \prime}+\cdots+\Delta_{n}^{-k} \mu_{2 n-1} D_{2 n-1}^{\prime \prime}$, where $D_{i}^{\prime \prime}=D_{i}+\frac{1}{n-2} D_{n}$ for all $i \in \llbracket 1, n-1 \rrbracket \cup \llbracket n+1,2 n-1 \rrbracket$.

Hence

$$
\begin{aligned}
D\left(Y_{1,1}\right)= & \Delta_{n}^{-k} \operatorname{ad}_{x}\left(Y_{1,1}\right)+\Delta_{n}^{-k} \frac{1}{n-2}\left(\mu_{1}+\cdots+\mu_{n-1}\right. \\
& \left.+\mu_{n+1}+\cdots+\mu_{2 n-1}\right) Y_{1,1}, \\
D\left(Y_{1, \alpha}\right)= & \Delta_{n}^{-k} \operatorname{ad}_{x}\left(Y_{1, \alpha}\right)+\Delta_{n}^{-k} \mu_{n+1-\alpha} Y_{1, \alpha} \quad \text { for } \alpha \geq 2, \\
D\left(Y_{i, 1}\right)= & \Delta_{n}^{-k} \operatorname{ad}_{x}\left(Y_{i, 1}\right)+\Delta_{n}^{-k} \mu_{n+i-1} Y_{i, 1} \quad \text { for } i \geq 2,
\end{aligned}
$$

and

$$
\begin{aligned}
D\left(Y_{i, \alpha}\right)= & \Delta_{n}^{-k} \operatorname{ad}_{x}\left(Y_{i, \alpha}\right)+\Delta_{n}^{-k}\left(\mu_{n+1-\alpha}+\mu_{n+i-1}\right. \\
& \left.\quad-\frac{1}{n-2}\left(\mu_{1}+\cdots+\mu_{n-1}+\mu_{n+1}+\cdots+\mu_{2 n-1}\right)\right) Y_{i, \alpha}
\end{aligned}
$$


when $i \geq 2$ and $\alpha \geq 2$.

Set $\bar{y}:=\varphi^{-1}(x)$, and write $y=\sum_{l \in \mathbb{Z}} y_{l} t^{l}$ with $y_{l} \in \mathcal{O}_{q}\left(\mathrm{SL}_{n}\right)$ equal to 0 except for a finite number of values of $l$. Also, for all $i \in \llbracket 1, n-1 \rrbracket \cup \llbracket n+1,2 n-1 \rrbracket$, we set $\varphi^{-1}\left(\mu_{i}\right)=\sum_{l \in \mathbb{Z}} \mu_{i, l} t^{l}$ with $\mu_{i, l} \in \mathcal{O}_{q}\left(\mathrm{SL}_{n}\right)$ equal to 0 except for a finite number of values of $l$. Now $\varphi^{-1}\left(\mu_{i}\right)$ is central in $\mathcal{O}_{q}\left(\mathrm{SL}_{n}\right)\left[t^{ \pm 1}\right]$, since $\mu_{i}$ is central in $\mathcal{O}_{q}\left(M_{n}\right)$; and so $\varphi^{-1}\left(\mu_{i}\right) \in K\left[t^{ \pm 1}\right]$. Hence $\mu_{i, l} \in K$, for all $i, l$. Then, straightforward computations show that

$$
D^{\prime}=\operatorname{ad}_{y_{k}}+\mu_{1, k} D_{1}^{\prime}+\cdots+\mu_{n-1, k} D_{n-1}^{\prime}+\mu_{n+1, k} D_{n+1}^{\prime}+\mu_{2 n-1, k} D_{2 n-1}^{\prime} .
$$

We show this when $(i, \alpha)=(1,1)$, the other cases are proved in a similar manner.

In this case, $D^{\prime}\left(X_{1,1}\right)=\varphi^{-1} \circ D\left(Y_{1,1} \Delta_{n}^{-1}\right)$, that is,

$$
\begin{aligned}
& D^{\prime}\left(X_{1,1}\right)=\varphi^{-1}\left(\Delta_{n}^{-k-1} \operatorname{ad}_{x}\left(Y_{1,1}\right)+\Delta_{n}^{-k-1} \frac{1}{n-2}\left(\mu_{1}+\cdots\right.\right. \\
& \left.\left.\cdots+\mu_{n-1}+\mu_{n+1}+\cdots+\mu_{2 n-1}\right) Y_{1,1}\right) \\
& =\sum_{l \in \mathbb{Z}}\left[\operatorname{ad}_{y_{l}}\left(X_{1,1}\right)+\frac{1}{n-2}\left(\mu_{1, l}+\cdots\right.\right. \\
& \left.\left.\cdots+\mu_{n-1, l}+\mu_{n+1, l}+\cdots+\mu_{2 n-1, l}\right) X_{1,1}\right] t^{l-k} .
\end{aligned}
$$

Now, as $\mathcal{O}_{q}\left(\mathrm{SL}_{n}\right)\left[t^{ \pm 1}\right]=\bigoplus_{l \in \mathbb{Z}} \mathcal{O}_{q}\left(\mathrm{SL}_{n}\right) t^{l}$ and $D^{\prime}\left(X_{1,1}\right) \in \mathcal{O}_{q}\left(\mathrm{SL}_{n}\right)$, we deduce from the previous equality that

$$
\begin{aligned}
D^{\prime}\left(X_{1,1}\right)= & \operatorname{ad}_{y_{k}}\left(X_{1,1}\right)+\frac{1}{n-2}\left(\mu_{1, k}+\ldots\right. \\
& \left.\quad \cdots+\mu_{n-1, k}+\mu_{n+1, k}+\cdots+\mu_{2 n-1, k}\right) X_{1,1} \\
=\operatorname{ad}_{y_{k}}\left(X_{1,1}\right)+\mu_{1, k} D_{1}^{\prime}\left(X_{1,1}\right)+\cdots+\mu_{n-1, k} D_{n-1}^{\prime}\left(X_{1,1}\right) & \quad+\mu_{n+1, k} D_{n+1}^{\prime}\left(X_{1,1}\right)+\cdots+\mu_{2 n-1, k} D_{2 n-1}^{\prime}\left(X_{1,1}\right),
\end{aligned}
$$

as desired.

To finish, let us mention that the decomposition of $D^{\prime}$ is unique because of the uniqueness of the decomposition of $D$ in $\mathcal{O}_{q}\left(\mathrm{GL}_{n}\right)$.

Note that the automorphism $\sigma$ of $\mathcal{O}_{q}\left(M_{n}\right)$ defined in the discussion before Corollary 2.10 induces an automorphism of $\mathcal{O}_{q}\left(\mathrm{SL}_{n}\right)$, still denoted by $\sigma$, since $\sigma\left(\Delta_{n}\right)=$ $\Delta_{n}$. Following the same reasoning as in the discussion before Corollary 2.10, we obtain the following result regarding the twisted Hochschild homology of $\mathcal{O}_{q}\left(\mathrm{SL}_{n}\right)$.

Corollary 3.4. $\mathrm{HH}_{n^{2}-2}\left(\mathcal{O}_{q}\left(\mathrm{SL}_{n}\right), \mathcal{O}_{q}\left(\mathrm{SL}_{n}\right)_{\sigma}\right)$ is a finite dimensional vector space of dimension $2 n-2$.

When $n=2$, the derivations $D_{1}-D_{3}$ and $D_{2}$ of $\mathcal{O}_{q}\left(M_{n}\right)$ satisfy $\left(D_{1}-D_{3}\right)\left(\Delta_{n}\right)=$ $0=D_{2}\left(\Delta_{n}\right)$. Hence, they induce two derivations of $\mathcal{O}_{q}\left(\mathrm{SL}_{2}\right)$ that are denoted by $D_{1}^{\prime}$ and $D_{2}^{\prime}$. Then, by using arguments similar to those in the proof of Theorem 3.3, one can prove the following result. 
Proposition 3.5. 1) Every derivation $D^{\prime}$ of $\mathcal{O}_{q}\left(\mathrm{SL}_{2}\right)$ can be uniquely written as follows

$$
D^{\prime}=\operatorname{ad}_{y}+\mu_{1}^{\prime} D_{1}^{\prime}+\mu_{2}^{\prime} D_{2}^{\prime},
$$

with $\operatorname{ad}_{y} \in \operatorname{InnDer}\left(\mathcal{O}_{q}\left(\mathrm{SL}_{2}\right)\right)$ and $\mu_{1}^{\prime}, \mu_{2}^{\prime} \in Z\left(\mathcal{O}_{q}\left(\mathrm{SL}_{2}\right)\right)=K$.

2) $\mathrm{HH}^{1}\left(\mathcal{O}_{q}\left(\mathrm{SL}_{2}\right)\right)$ is a two-dimensional vector space with basis $\left(\overline{D_{1}^{\prime}}, \overline{D_{2}^{\prime}}\right)$.

3) $\mathrm{HH}_{2}\left(\mathcal{O}_{q}\left(\mathrm{SL}_{2}\right), \mathcal{O}_{q}\left(\mathrm{SL}_{2}\right)_{\sigma}\right)$ is a two-dimensional vector space.

Notice that Hadfield and Krähmer have computed the twisted Hochschild homology of $\mathcal{O}_{q}\left(\mathrm{SL}_{2}\right)$ in [6]. However, there is a misprint in [6, Theorem 1.1] in the dimension of $\mathrm{HH}_{2}\left(\mathcal{O}_{q}\left(\mathrm{SL}_{2}\right),{ }_{\sigma^{-1}} \mathcal{O}_{q}\left(\mathrm{SL}_{2}\right)\right) \simeq \mathrm{HH}_{2}\left(\mathcal{O}_{q}\left(\mathrm{SL}_{2}\right), \mathcal{O}_{q}\left(\mathrm{SL}_{2}\right)_{\sigma}\right)$, as the authors have confirmed.

\section{References}

[1] J.Alev and M. Chamarie, Dérivations et automorphismes de quelques algèbres quantiques. Comm. Algebra 20 (1992), 1787-1802. Zbl 0760.17003 MR 1162608

[2] K. A. Brown and J. J. Zhang, Dualising complexes and twisted Hochschild (co)homology for noetherian Hopf algebras. Preprint 2006, arXiv:Math.RA/0603732

[3] G. Cauchon, Effacement des dérivations et spectres premiers des algèbres quantiques. $J$. Algebra 260 (2003), 476-518. Zbl 1017.16017 MR 1967309

[4] G. Cauchon, Spectre premier de $O_{q}\left(M_{n}(k)\right)$ : image canonique et séparation normale. $J$. Algebra 260 (2003), 519-569. Zbl 1024.16001 MR 1967310

[5] P. Feng and B. Tsygan, Hochschild and cyclic homology of quantum groups. Comm. Math. Phys. 140 (1991), 481-521. Zbl 0743.17020 MR 1130695

[6] T. Hadfield and U. Krähmer, Twisted homology of quantum SL(2). K-Theory 34 (2005), 327-360. Zbl 1098.58006 MR 2242563

[7] T. Hadfield and U. Krähmer, On the Hochschild homology of quantum SL(N). C. R. Acad. Sci. Paris Sér. I Math. 343 (2006), 9-13. Zbl 05046013 MR 2241950

[8] A. Klimyk and K. Schmüdgen, Quantum groups and their representations. Texts Monogr. Phys., Springer-Verlag, Berlin 1997. Zbl 0891.17010 MR 1492989

[9] J. Kustermans, G. J. Murphy, and L. Tuset, Differential calculi over quantum groups and twisted cyclic cocycles. J. Geom. Phys. 44 (2003), 570-594. Zbl 1029.46114 MR 1943179

[10] S. Launois and T. H. Lenagan, Primitive ideals and automorphisms of quantum matrices. Algebr. Represent. Theory 10 (4), 339-365 ; arXiv:math.RA/0511409

[11] T. Levasseur and J. T. Stafford, The quantum coordinate ring of the special linear group. J. Pure Appl. Algebra 86 (1993), 181-186. Zbl 0784.17023 MR 1215645

[12] J. M. Osborn and D. S. Passman, Derivations of skew polynomial rings. J. Algebra 176 (1995), 417-448. Zbl 0865.16020 MR 1351617 
[13] B. Parshall and J. P. Wang, Quantum linear groups. Mem. Amer. Math. Soc. 89 (1991). Zbl 0724.17011 MR 1048073

[14] M. Van den Bergh, A relation between Hochschild homology and cohomology for Gorenstein rings. Proc. Amer. Math. Soc. 126 (1998), 1345-1348; erratum ibid. 130 (2002), 2809-2810. Zbl 0894.16005 MR 1443171 MR 1900889

Received September 13, 2006

S. Launois, Institute of Mathematics, Statistics and Actuarial Science, University of Kent at Canterbury, CT2 7NF, UK

E-mail: stephane.launois@ed.ac.uk

T. H. Lenagan, Maxwell Institute for Mathematical Sciences, School of Mathematics, University of Edinburgh, James Clerk Maxwell Building, King's Buildings, Mayfield Road, Edinburgh EH9 3JZ, Scotland

E-mail: tom@maths.ed.ac.uk 